\title{
V. Sowjetische Eingriffe in das Normensystem und die Anfänge der Justizsteuerung durch die DJV (1945-1947)
}

\section{Eingriffe in die Wirtschaftsstrafjustiz}

Trotz allgemeiner Zurückhaltung der Besatzungsmacht bei Umwandlung des deutschen Normensystems in den Jahren 1945 bis 1947 aufgrund übergeordneter pragmatischer und deutschlandpolitischer Überlegungen lassen sich im Wirtschaftsstrafrecht wie in dem dazu gehörigen Prozeßrecht bereits seit 1945/46 sowjetische Interventionen ausmachen. Eingriffe auf diesem Gebiet lagen besonders nahe, ging es doch darum, die zerstörte deutsche Wirtschaft wieder in Gang zu setzen und Wirtschaftsverbrechen möglichst effektiv zu verfolgen. Auch in den Westzonen und in Berlin wiesen die Besatzungsmächte die deutschen Gerichte immer wieder zu strenger Bestrafung von Wirtschaftsstraftaten an ${ }^{1}$, so daß rigorose Maßnahmen auf diesem Gebiet allein nicht als Ausdruck einer spezifisch sowjetischen Transformationstendenz zu bewerten sind. Jedoch lassen sich im $\mathrm{Zu}$ sammenhang mit Befehl Nr. 160 vom 3. Dezember 1945, der auch im Gefolge dieses Befehls ergangenen Anweisung zur Abhaltung von Schauprozessen sowie durch die auf Verlangen der Besatzungsmacht erlassene Verordnung über das Verfahren in Wirtschaftsstrafsachen vom 21. Oktober 1947 deutlich die Tendenz zur Einführung sowjetischer Rechtsbegriffe und Justizpraktiken sowie die Durchbrechung deutscher rechtsstaatlicher Grundsätze erkennen.

Auch nach 1945 blieben zunächst die im Zweiten Weltkrieg erlassenen Strafbestimmungen wie die Kriegswirtschaftsstrafverordnung, die Verbrauchsregelungsstrafverordnung und die Preisstrafverordnung zur Ahndung von Wirtschaftsstraftaten weiter in $\mathrm{Kraft}^{2}$. Jedoch schienen diese Regelungen der SMAD zur Aburteilung schwerer wirtschaftlicher Straftaten, bei denen die Täter „absichtlich dem Wiederaufbau Schwierigkeiten " bereitet hatten, nicht auszureichen ${ }^{3}$. Daher erließ sie am 3. Dezember 1945 Befehl Nr. 160 ,über die Verantwortung für Sabotage- und Diversionsakte“. Der Originaltext des Befehls wurde nie veröffentlicht, so daß die Fassungen in den deutschen Gesetzes- und Verordnungsblättern der

1 Für Berlin siehe die Eintragungen in der Berliner Chronik, in: Berlin 1945-1946, S. 529 (13. 9. 1946), sowie in: Berlin 1946-1948, S. 174 (14.3. 1947), S. 195 (8. 4. 1947).

Vgl. u. a. Buchholz, Entwicklung, S. 602.

3 Siehe Vermerk Kuenzers, 30.11. 1945, über eine Mitteilung der SMAD-Rechtsabteilung, derzufolge auf der Insel Rügen "von offenbar volksfeindlichen Elementen“ Maschinen abmontiert, zerlegt und anschließend versenkt worden seien. Die Täter seien zwar gefaßt, "mangels angeblich entscheidender Strafbestimmungen" jedoch nicht bestraft worden, BAB, DP1 VA Nr. 2, Bl. $114 \mathrm{f}$. 
einzelnen Länder und Provinzen voneinander abwichen ${ }^{4}$. Inhaltlich stimmten sie indes darin überein, daß im ersten Absatz der dem deutschen Strafrecht fremde Begriff der Diversion als Durchkreuzung von wirtschaftlichen Maßnahmen der deutschen Selbstverwaltungsorgane oder der deutschen Verwaltungen und im zweiten Absatz Sabotage als Handlung bezeichnet wurde, die die Arbeit von Wirtschaftsbetrieben beeinträchtige bzw. zerstöre. Dabei handelte es sich Fritz Löwenthal zufolge, der über zwölf Jahre im sowjetischen Exil verbracht hatte, um eine Verwechslung dieser beiden Begriffe: Denn im Sowjetrecht sei Diversion „auf die Zerstörung wirtschaftlicher Werte oder die Vernichtung von Menschenleben“, Sabotage hingegen auf die Behinderung des „normalen Ablauf[s] der Tätigkeit öffentlicher Einrichtungen oder des Wirtschaftslebens" bezogen ${ }^{5}$. Daß hier eine Begriffsverwirrung vorlag, zeigt auch die Definition in der Direktive der DJV vom 19. Dezember 1945: „Unter Diversionsakten ist zu verstehen: Vorsätzliche Zerstörung oder Beschädigung - sei es durch Sprengung, durch Brandstiftung oder auf irgend eine andere Weise - von Eisenbahnen und anderen Verbindungs- und Verkehrswegen und -mitteln, Wasserleitungen, Fabriken und ihren Einrichtungen, öffentlichen Lägern [sic] und anderen Einrichtungen, oder öffentlichen oder für die Öffentlichkeit wichtigen Vermögen. Unter Sabotage ist zu verstehen: Vorsätzliche Nichterfüllung bestimmter Pflichten oder bewußt nachlässige Erfüllung dieser Pflichten."6 Auch die Äußerung von Oberstleutnant Lyssjak auf der Länderkonferenz vom 11. März 1946 stimmt nicht mit dem Befehlstext, sondern mit der zitierten Definition überein: „Vernichtung von landwirtschaftlichen Maschinen ist ein Diversionsakt."7 Der Befehl schrieb sowohl bei Sabotage als auch bei Diversionsakten Freiheitsstrafen bis zu 15 Jahren und in besonders schweren Fällen sogar die Todesstrafe vor.

Auch bei der Durchführung von Befehl Nr. 160 fungierte die DJV als Hilfsorgan der SMAD. Denn sie wurde beauftragt, die zuständigen Gerichte zu benennen und die Kontrolle über die Durchführung des Befehls zu übernehmen ${ }^{8}$. In einer Direktive vom 19. Dezember 1945 übertrug sie den Strafkammern der Landgerichte die Zuständigkeit und forderte von den Generalstaatsanwälten Berichte über Einleitung der Verfahren, Anklageerhebungen und Ergebnisse der Prozesse9. Für die deutschen Justizbehörden wurde die DJV durch ihre im Befehlstext niedergelegte Funktion zum zentralen Ansprechpartner bei Verständnis- und Auslegungsfragen. Wie aus einer Anfrage der mecklenburgischen Justizverwaltung vom 18. Dezember hervorgeht, bereitete dort die sowjetische Begrifflichkeit erhebliche Schwierigkeiten, so daß um die authentische Übersetzung des Begriffs „Diversionshandlungen" gebeten wurde ${ }^{10}$. Die DJV antwortete zwar umgehend, ließ sich

4 Siehe Schuller, Geschichte, S. 8-10.

5 Löwenthal, Geist, S. 153.

6 Chef der DJV an Oberlandesgerichtspräsidenten und Generalstaatsanwälte, 19.12. 1945, in: Gesetze des Kontrollrats. Befehle der SMA, S. 45. Das Aktenexemplar der Direktive enthält diese Definition nicht (BAB, DP1 SE Nr. 2221, Bl. 73f.)

7 Protokoll der Länderkonferenz vom 11. 3. 1946, BAB, DP1 VA Nr. 839, Bl. 207.

8 Siehe Abs. 3 und 4 von Befehl Nr. 160, in: Gesetze des Kontrollrats. Befehle der SMA, S. 44 f.

9 Chef der DJV an Oberlandesgerichtspräsidenten und Generalstaatsanwälte, 19. 12. 1945, ebenda, S. 45 .

10 Abt. Justiz Mecklenburg-Vorpommern an DJV, 18. 12. 1945, BAB, DP1 SE Nr. 2221, Bl. 75. 
interessanterweise jedoch auch nicht auf eine Übersetzung des Begriffs ein, sondern beschränkte sich darauf, den nach Ziffer 1 und 2 des Befehls Nr. 160 unter Strafandrohung gestellten Tatbestand zu skizzieren ${ }^{11}$. Im übrigen ging es der DJV darum, die im Zusammenhang mit dem Befehl anfallenden Rechtsfragen zu klären, die sich auf die Geltungsdauer, die Zulässigkeit von Schnellverfahren und die Abgrenzung bzw. Vereinbarkeit mit anderen Strafnormen bezogen. Dabei verfügte die DJV über keinerlei eigenen Handlungspielraum. So konnte sie erst nach einer entsprechenden Mitteilung der SMAD-Rechtsabteilung am 15. Januar 1946 bekanntgeben, daß dem Befehl keine rückwirkende Kraft zukam ${ }^{12}$. Da sie auf die Anfrage bezüglich der Möglichkeit von Schnellverfahren ${ }^{13}$ keine Antwort erhielt, blieben ihr hier die Hände gefesselt.

Die Frage, ob Befehl Nr. 160 ausschließliche Bedeutung zugesprochen werden müsse, stellte sich, da die Landesverwaltung Mecklenburg auf Anordnung der dortigen SMA am 30. November 1945 ebenfalls eine Verordnung zur Bestrafung von Sabotagehandlungen erlassen hatte ${ }^{14}$, die, so Schiffer, ,in mehreren Punkten über den Befehl Nr. 160 hinaus“ ging. Gegenüber Karlshorst vertrat er die Auffassung, daß die Länder und Provinzen sich zwar strikt an den Befehl halten müßten, „daß aber gleichwohl der Erlaß ergänzender Strafbestimmungen im Rahmen des Notwendigen nicht ausgeschlossen" sei ${ }^{15}$. Die Frage wurde auf Antrag der DJV auf der Länderkonferenz vom 11. März 1946 beraten. Ihre Beantwortung erfolgte freilich nicht durch die Diskussion, sondern durch die autoritative Äußerung von Oberstleutnant Lyssjak, der kritisierte, daß der mecklenburgischen Verordnung ein falscher Sabotagebegriff zugrundegelegt worden sei. Weiterer Handlungsbedarf bestand jedoch nicht, da Ministerialdirektor Wilhelm Heinrich aus Schwerin berichtete, die Sabotageverordnung sei nach Erlaß von Befehl Nr. 160 aufgehoben worden.

Im Verlauf der Diskussion, in der der Tatbestand der Gerüchtemacherei ${ }^{16}$ mit dem der Sabotage teilweise gemeinsam behandelt wurde, sahen sich die SMADVertreter Lyssjak und Nikolajew zu einer klärenden Stellungnahme zu Befehl Nr. 160 veranlaßt. Zum einen stellten sie fest, daß Gerüchtemacherei keineswegs als Sabotage anzusehen sei, und zum anderen, daß sowohl Sabotage als auch Diversionsakte nur vorsätzlich begangen werden könnten und die Wirtschaft „reell“ beeinträchtigen müßten. „Um einer falschen Auslegung des Befehls vorzubeugen“, so Lyssjak, „sollte eine Instruktion ausgearbeitet werden, um die nötigen

1 Chef der DJV an Präsident von Mecklenburg-Vorpommern/Abt. Justizverwaltung, 20.12. 1945, ebenda, Bl. 76.

12 SMAD-Rechtsabteilung an Chef der DJV, 11. 1. 1946, BAB, DP1 SE Nr. 2221, Bl. 80; Weisung der DJV an Landes- und Provinzialverwaltungen, 15.1. 1946, ebenda, Bl. 78; gedruckt in: Gesetze des Kontrollrats. Befehle der SMA, S. 45.

13 Chef der DJV an SMAD-Rechtsabteilung, 18. 4. 1946, ebenda, Bl. 109; vgl. auch Vemerk Winkelmanns, 27. 1. 1947, BAB, DP1 VA Nr. 5930.

14 Verordnung Nr. 43, Bestrafung von Sabotagehandlungen, 30.11. 1945, in: Faschismus. Falsche und echte Befreier, S. $219 \mathrm{f}$. Auf Anfrage vom 19. 12. 1945 hatte die Justizverwaltung in Mecklenburg-Vorpommern am 14. 1. 1946 die Verordnung, deren Erlaß und Fassung „auf Anordnung und Wünsche der hiesigen Sowjetischen Militär-Administration" zurückgehe, der DJV übersandt, BAB, DP1 SE Nr. 2221, Bl. $63 \mathrm{f}$.

15 Chef der DJV an SMAD-Rechtsabteilung, 23. 2. 1946, BAB, DP1 VA Nr. 6957, Bl. $4 \mathrm{f}$.

16 Siehe dazu Kap. A II.3. 
Richtlinien und Klarheiten in dieser Frage zu schaffen." 17 Die DJV reichte daraufhin am 22. März in Karlshorst einen Instruktionsentwurf ein, in dem als erste Voraussetzung zur Anwendung des Befehls angeführt wurde, „daß der Täter gehandelt hat zu dem Zwecke, durch Sabotage- oder Diversionsakte den [...] Wiederaufbau zu schädigen“. Falls also eine entsprechende Absicht vorliege, könnten Straftaten wie Schwarzschlachten, die sonst mit anderen Strafvorschriften bedroht seien, zu Verstößen gegen Befehl Nr. 160 werden ${ }^{18}$. Dieser Instruktionsentwurf, der die Anforderungen der SMAD-Rechtsabteilung erfüllte, wurde in Karlshorst zwar gebilligt; jedoch war man sich Anfang Mai 1946 dort noch unklar, ob eine eigene Instruktion erlassen oder die der DJV bestätigt werden sollte ${ }^{19}$.

Warum eine Weisung ausblieb, läßt sich nicht klären und erscheint angesichts des großen Interesses der SMAD an der korrekten Durchführung des Befehls unverständlich. Denn die Rechtsabteilung revidierte die Justizbehörden in Mecklenburg im Hinblick auf die Durchführung von Befehl Nr. 160 und deckte nach ihren eigenen Worten dort „Fälle grober Verletzung des Befehls“ auf. In 123 Fällen von 372 zwischen Januar und April 1946 verhandelten Strafsachen hätten die Gerichte befehlswidrig Geldstrafen verhängt. „Dieser Umstand zeigt“, so Karassjow in einem Schreiben an Schiffer vom 15. Juni, „daß die Gerichte entweder bewußt gegen den Befehl Nr. 160 verstoßen, oder aber nach diesem Befehl solche Taten qualifizieren, welche wegen ihrer Geringfügigkeit nicht unter den Befehl fallen können, sondern nach anderen Gesetzesbestimmungen zu qualifizieren sind. Ich bitte, Maßnahmen zur Beseitigung von Fällen der Verletzung des Befehls Nr. 160 vom 3. Dezember 1945 seitens der Gerichte zu ergreifen."20 Am selben Tag ordnete Jakupow an, einen Bericht über alle vom 1. Januar bis 1. Juni 1946 aufgrund von Befehl Nr. 160 gefällten Urteile anzufertigen ${ }^{21}$. Wie genau die Rechtsabteilung informiert werden wollte, zeigt die Weitergabe dieser Anweisung an die Ländervertreter: „Es käme insbesondere auch darauf an, ob die Strafanträge und die Urteile sich im wesentlichen entsprochen hätten, die Strafhöbe angemessen sei, und alle Mittel zur Bekämpfung der Kriminalität, insbesondere durch Heranziebung der Presse, in Betracht gezogen seien. [...] Der SMA sei daran gelegen, festzustellen, ob einzelne Richter versagt hätten." 22 Mit derartigen Informationen sollten offensichtlich die Grundlagen für Anleitungsstrukturen im Justizwesen gelegt werden, die die richterliche Unabhängigkeit und damit die Rechtsstaatlichkeit beeinträchtigen konnten.

Da die SMAD-Rechtsabteilung sich nicht auf Berichte der DJV verließ, sondern auch weiterhin selbst die Gerichte revidierte, stellte sie, gerade im Hinblick auf die Durchführung von Befehl Nr. 160, auch weiterhin gravierende Mängel

17 Protokoll der Länderkonferenz vom 11. 3. 1946, BAB, DP1 VA Nr. 839, Bl. $207 \mathrm{f}$.

18 Chef der DJV an Chef der SMAD-Rechtsabteilung, 22. 3. 1946, und Entwurf einer „Instruktion über die Auslegung des Befehls Nr. 160“, BAB, DP1 SE Nr. 2221, Bl. 102-104 (Hervorhebung im Original).

19 Mitteilung Melsheimers in: Protokoll der Länderkonferenz vom 3.5. 1946, BAB, DP1 VA Nr. 22, Bl. 1.

20 Chef der SMAD-Rechtsabteilung an Chef der DJV, 15. 6. 1946, BAB, DP1 SE Nr. 2221, Bl. 114.

21 Aktenvermerk über eine Rücksprache mit Jakupow am 15. 6. 1946, ebenda, Bl. 110.

22 Protokoll der Länderkonferenz vom 14./15. Juni 1946, BAB, DP1 VA Nr. 22, Bl. 29, Hervorhebungen im Original. 
fest. Nach einer zehntägigen Revision der Gerichte in Brandenburg im Juli 1946 hielt Jakupow fest, daß dort teilweise Strafsachen nach Befehl Nr. 160 von den Amtsgerichten - und nicht, wie es seit dem 19. Dezember 1945 vorgeschrieben war, von den Strafkammern der Landgerichte - abgeurteilt worden seien. Besonders kritisierte die sowjetische Seite, daß die Justizverwaltung davon Kenntnis gehabt habe, aber nicht eingeschritten sei23. Dabei waren die beanstandeten Fehler nicht durchweg auf Unzulänglichkeiten der deutschen Gerichte zurückzuführen, sondern auch auf örtliche sowjetische Kommandanten, die den Gerichten oftmals befahlen, in Fällen, die sie für Sabotage hielten, Verfahren nach Befehl Nr. 160 durchzuführen. Die sowjetischen Offiziere nahmen wenig Rücksicht auf die rechtliche Ausgestaltung des Befehls, indem sie etwa auf Aburteilungen „in Schauprozessen durch die Schöffengerichte" drängten. Ein Bericht der Potsdamer Justizverwaltung vom 19. Juli 1946 vermerkte in diesem Zusammenhang: „Anklagevertreter und Richter, die sich diesem Verlangen widersetzen, haben mit erheblichen Schwierigkeiten zu rechnen." 24

Vor dem Hintergrund der sowjetischen Beanstandungen ${ }^{25}$ und der Berichterstattung an die DJV wurde die Instruktion zur Durchführung von Befehl Nr. 160 gegenüber der ursprünglich im März bei der SMAD-Rechtsabteilung eingereichten Fassung stark erweitert ${ }^{26}$. Die Rundverfügung vom 23. August 1946 unterschied zwischen dem äußeren und dem inneren Tatbestand ${ }^{27}$. Auf die sowjetische Weisung vom 15. Juni 1946 unmittelbar Bezug nehmend, ordnete sie an, bei der Beurteilung des äußeren Tatbestands zu beachten, daß nur dann nach Befehl Nr. 160 zu verfahren sei, wenn Verstöße gegen Verwaltungsanordnungen „den wirtschaftlichen Aufbau mehr als geringfügig beeinträchtigt" hätten. Auch das in diesem Zusammenhang angeführte ausdrückliche Verbot, Geldstrafen zu verhängen, war auf die Kritik der SMAD-Rechtsabteilung zurückzuführen. Der innere Tatbestand erforderte nach der Rundverfügung Böswilligkeit, was nicht nur vorsätzliches Handeln implizierte. Der „böse Wille“ des Täters komme insbesondere dann zum Ausdruck, „wenn er durch feindselige Einstellung gegenüber dem demokratischen Aufbau, aber auch dann, wenn er durch Habgier, Trotz oder zur Förderung fremder Gewinnsucht zu seinem Verhalten bestimmt worden" sei. Damit hatte die Rundverfügung die ursprüngliche sowjetische Leitlinie vom März aufgegriffen, daß nur absichtsvolles Handeln zu bestrafen sei, war aber insofern über den Instruktionsentwurf der DJV hinausgegangen, als die Absicht sich nicht nur auf die Schädigung des Wiederaufbaus beziehen mußte. Dies hatte Jakupow bereits in einer Unterredung mit Schiffer, Melsheimer und Rosenthal-Pelldram

23 Aktenvermerk über den Besuch Schiffers in Karlshorst am 31.7. 1946, BAB, DP1 VA Nr. 11, Bl. $62 \mathrm{f}$.

${ }_{24} \mathrm{Zu}$ den sowjetischen Eingriffen in die Gerichtstätigkeit nach Befehl Nr. 160 siehe Löwenthal, Geist, S. 155-160; das Zitat S. 156.

${ }_{25}$ Zur entsprechenden sowjetischen Gerichtskritik und Einflußnahme in Brandenburg und Thüringen vgl. Pohl, Justiz in Brandenburg (Manuskript), S. 60f. und Weber, Justiz und Diktatur, S. $80 \mathrm{f}$.

$26 \mathrm{Daß}$ vor allem die sowjetische Kritik im Hintergrund der Rundverfügung vom 23. 8. 1946 stand, wird von Benjamin u. a., Geschichte der Rechtspflege 1945-1949, S. 246, nicht angeführt.

27 DJV an Präsidenten der Landes- und Provinzialverwaltungen/Abteilung Justiz, 23. 8. 1946, BAB, DP1 VA Nr. 323, Bl. 11-13; dazu auch Benjamin u.a., Geschichte der Rechtspflege 1945-1949, S. 246. 
am 15. Juni 1946 verdeutlicht ${ }^{28}$, so daß auch diese Weiterung auf eine sowjetische Anordnung zurückging. Schließlich wies die Rundverfügung darauf hin, daß auch andere Befehle der SMAD bei ihren Strafbestimmungen auf Befehl Nr. 160 Bezug nähmen ${ }^{29}$.

Trotz dieser Anweisung blieb, wie aus einem Schreiben Bukanows an Schiffer vom 2. Oktober 1946 hervorgeht, die Rechtsprechung nach Befehl Nr. 160 nach Auffassung der SMAD-Rechtsabteilung unbefriedigend. Diese kritisierte erstens, daß die Richter und Staatsanwälte „in Unkenntnis der wirtschaftlich-politischen Zustände [...] in formal-bürokratischer Weise" Recht sprächen. Zweitens verbleibe die Gerichtspraxis trotz wiederholter Hinweise der SMAD-Rechtsabteilung, daß Sabotage und Diversion hart zu bestrafen seien, „äußerst liberal“. Drittens würden Verstöße gegen die Befehle Nr. 71 (vom 6. März 1946), 163 (vom 27. Mai 1946) und 171 (17. Juni 1946), die sich auf die Pflichtablieferung von landwirtschaftlichen Produkten bezogen, trotz der in ihnen genannten Vorschrift nicht nach Befehl Nr. 160 bestraft. Viertens schließlich dauerten Voruntersuchungen und Hauptverhandlungen in Strafsachen nach Befehl Nr. 160 teilweise bis zu sechs Monaten, was zu lang sei. Darauf folgte eine Reihe von Forderungen für die „Verbesserung“ der Rechtsprechung 30 . Sowohl aus dem Schreiben Bukanows als auch aus Darlegungen Jakupows auf der Länderkonferenz vom 1./2. November 1946 ging hervor, daß die SMAD-Rechtsabteilung insbesondere die Effektivität der Rechtsprechung nach Befehl Nr. 160 steigern wollte: „Mit einem Prozeß“, so Jakupow, "müssen große Resultate erzielt werden.“ Dabei komme es darauf an, die Durchführung der wirtschaftlichen Maßnahmen in dem betreffenden Bezirk zu berücksichtigen und "die richtige Repression danach [zu] richten“. Die Prozesse sollten nicht nur die Hauptverantwortlichen streng bestrafen, sondern auch zur Vorbeugung weiterer Sabotagefälle dienen. Dazu, so die schriftliche Anweisung, müßten sorgsam ausgewählte Fälle ,in öffentlichen Gerichtssitzungen unter weiter Heranziehung der Bevölkerung und Beleuchtung in der Presse" - also in Schauprozessen - behandelt werden. Hauptziel war somit neben der Ahndung von Verbrechen auch die Erziehung und Abschreckung der Bevölkerung. Schließlich ordnete die SMAD-Rechtsabteilung an, die Voruntersuchung in den Sabotage- und Diversionssachen in zehn und die Gerichtsverhandlungen in fünf Tagen durchzuführen ${ }^{31}$.

So engagiert die sowjetische Seite darin war, die Durchführung von Befehl Nr. 160 in ihrem Sinne voranzutreiben, so wenig entgegenkommend zeigte sie sich gegenüber den Wünschen der Vertreter der deutschen Justizverwaltungen. Diese meldeten keinen Widerspruch gegen die sowjetischen Forderungen an, sahen aber angesichts der steigenden Anzahl der Fälle - die SMA in Potsdam hatte nach dem Bericht von Hoeniger festgestellt, „daß ganze Dörfer unter Billigung ihrer Bürgermeister ihr Ablieferungssoll nicht erfüllt hätten" - keine Chance zur

28 Aktenvermerk über eine Rücksprache mit Jakupow am 15.6. 1946, BAB, DP1 SE Nr. 2221, Bl. 110.

29 Eine genaue Aufstellung dieser SMAD-Befehle, die sich alle auf Ablieferpflichten von landwirtschaftlichen Produkten beziehen, bei Ristow, Probleme, S. 126.

30 SMAD-Rechtsabteilung an Chef der DJV, 2. 10. 1946, BAB, DP1 SE Nr. 2221, Bl. $128 \mathrm{f}$.

31 Ebenda und Protokoll der Länderkonferenz vom 1./2. 11. 1946, BAB, DP1 VA Nr. 22, Bl. 84-86, Hervorhebung im Original. 
Einhaltung der Fristen, wenn die Sachen nach Befehl Nr. 160 ausschließlich von den Landgerichten abgeurteilt werden durften. Schiffers Bitte, die leichteren Fälle an das Schöffengericht überweisen zu dürfen, entsprach Jakupow nicht. Er argumentierte streng juristisch und verwies auf die allgemeine Bestimmung, „daß Sabotagefälle von den Strafkammern abzuurteilen" seien; dem Amtsgericht - das Schöffengericht nannte er nicht - stehe es nicht zu, hohe Gefängnisstrafen und die Todesstrafe anzuwenden. Seine zentrale Aussage lautete jedoch: „Wir können nicht den Befehl des obersten Chefs abändern und müssen daher die Sache erst überdenken." 32 Das Verhalten der SMAD-Rechtsabteilung war somit widersprüchlich: Einerseits führte sie im Zusammenhang mit Befehl Nr. 160 sowjetische Rechtsbegriffe ein und versuchte, die Rechtsprechung in ihrem Sinne zu steuern, während sie andererseits an den Zuständigkeitsregelungen des deutschen Gerichtsverfassungsrechts festhielt.

Die DJV gab die Weisungen vom 2. Oktober am 3. Dezember 1946 zwar unverändert an die Landesjustizabteilungen weiter ${ }^{33}$; die Erfolge dieses ersten Versuchs, die Justiz in einem für die Besatzungsmacht offensichtlich zentralen Punkt zu lenken, blieben jedoch äußerst begrenzt. Denn Jakupow sah sich genötigt, auf einer Tagung der Generalstaatsanwälte am 25./26. April 1947 seine Kritik an der seines Erachtens mangelhaften Rechtsprechung und zu milden Bestrafung von Verbrechen nach Befehl Nr. 160 zu wiederholen ${ }^{34}$. Auch die überlieferten Zahlen sprechen eine deutliche Sprache. Einem Bericht vom 28. August 1948 zufolge wurden zwischen April und Dezember 1946 insgesamt 488 Personen gemäß Befehl Nr. 160 verurteilt, davon 119 zu Geldstrafen und 118 zu Gefängnisstrafen unter drei Monaten. Im Jahre 1947 waren es insgesamt 647 Personen, aber allen Anwei $i_{\bar{T}}$ sungen zum Trotz hatten die Gerichte 202 von ihnen zu Geldstrafen und $110 \mathrm{zu}$ Gefängnisstrafen unter drei Monaten verurteilt ${ }^{35}$.

Nicht nur die Umsetzung von Befehl Nr. 160 bereitete den Deutschen Schwierigkeiten, sondern auch die Durchführung von Strafprozessen vor erweiterter Öffentlichkeit. Diese Prozeßform, besser bekannt als Schauprozeß, war in der Sowjetunion ein gängiges Mittel, um eine abschreckende Wirkung unter den $\mathrm{Zu}$ schauern zu erzielen; in Deutschland hingegen war sie weitgehend unbekannt. Schauprozesse waren in der SBZ auf lokaler Ebene von den sowjetischen Kommandanten bereits eingeführt worden ${ }^{36}$. Sie dienten zwar nicht ausschließlich, jedoch zu einem großen Teil der Aburteilung von Wirtschaftsstraftaten und waren bis April 1947 bereits so etabliert, daß auch die Landesregierungen, die Generalstaatsanwälte oder einzelne Gerichte die Abhaltung von Schauprozessen anordneten $^{37}$. Ihre Ergebnisse entsprachen freilich nicht immer den an sie gestellten Er-

32 Ebenda, Bl. 83, 86.

33 DJV an die Justizabteilungen in Schwerin, Dresden, Weimar, Potsdam, Halle, 3.12. 1946, BAB, DP1 VA Nr. 323, Bl. $48 \mathrm{f}$.

34 Protokoll der Konferenz der Generalstaatsanwälte am 25./26. 4. 1947, BAB, DP1 VA Nr. 14, Bl. 77-80.

35 DJV an SMAD-Rechtsabteilung, 28. 8. 1948, Betr.: Geschäftsentwicklung bei den Gerichten und Staatsanwaltschaften [vom 1. 1. 1946 bis 30.6. 1948], BAB, DP1 VA Nr. 6196, Bl. 7.

$36 \mathrm{Vgl}$. Löwenthal, Geist, S. $174 \mathrm{f}$.

37 Vermerk Ackermanns, 11. 9. 1947, über die Erfahrungen mit der Durchführung größerer Schauprozesse, BAB, DP1 VA Nr. 347, Bl. 33f. Dem Vermerk nach handelte es sich bei 14 von 23 bis 
wartungen. Insbesondere spielte das Publikum oft nicht die ihm zugedachte Rolle, sondern reagierte auf die Verhandlung und deren Ergebnis mit Unmutsbekundungen. Dies war unter anderem im Januar 1947 der Fall bei einem Schauprozeß in Magdeburg gegen Zwangsdienstverpflichtete, die ihre Arbeit - die Demontage einer Fabrik - verlassen hatten. Im Verlauf des Verfahrens beklagten diese sich über die Unterbringung in ungeheizten Scheunen, unzureichende Verpflegung und rücksichtslose Antreiberei. Als der vorsitzende Richter ihnen ins Wort fiel, entstand im Zuschauerraum erhebliche Unruhe, und die Verhandlung konnte nur mit Mühe zu Ende geführt werden. Die SMAD-Rechtsabteilung erfuhr davon und beauftragte am 3. April 1947 die DJV mit der Untersuchung des Falles. Dabei stellte sich heraus, daß der Kreiskommandant den Prozeß überstürzt angeordnet hatte ${ }^{38}$; die Verantwortung für das Verfahren lag somit nicht bei der deutschen, sondern bei der sowjetischen Seite. Kurz darauf, am 16. Mai, stellte der Leiter der Abteilung III, Fritz Löwenthal, auf einer Dienstbesprechung der DJV fest, daß Schauprozesse "bisweilen nicht unzweckmäßig" seien, sie jedoch oft - wie in Magdeburg - in ungeeigneter Form durchgeführt würden. Ebenfalls abzulehnen sei die Durchführung von Schauprozessen im Kino mit von der Staatsanwaltschaft vertriebenen Eintrittskarten. Schiffer pflichtete Löwenthal bei, indem er zwei Vorbedingungen für einen Schauprozeß nannte: Zum einen müsse das Ergebnis des Verfahrens feststehen und zum anderen „müsse der Vorsitzende ein besonders ausgesuchter Richter sein“. „Schauprozesse“, so fuhr er fort, "seien nicht zur Befriedigung des Sensationsbedürfnisses da. Auch die Unabhängigkeit der Rechtspflege könne dadurch beeinträchtigt werden" 39 . Mit der Auffassung der SMAD-Rechtsabteilung stimmten Schiffer und Löwenthal nur insoweit überein, daß derartige Verfahren nur bei guter Vorbereitung und mit feststehendem Ausgang sinnvoll seien ${ }^{40}$; im Unterschied zu den Sowjets waren diese und andere Vertreter rechtsstaatlichen Denkens mit dem Oberlandesgerichtspräsidenten von Halle, Hans-Diedrich Schmidt, überwiegend der Meinung, „daß die Schauprozesse nicht der Würde des Gerichts entsprechen und nicht geeignet sind, das Ansehen der Justiz zu heben“. Trotz aller Einwände und der Schlußfolgerung, „bei Anberaumung von Schauprozessen größte Zurückhaltung " ${ }^{41}$ zu wahren, konnten die Vertreter der DJV jedoch letztlich den weiteren Einsatz dieser Prozeßform nicht verhindern.

Die sowjetischen Eingriffe in die Wirtschaftsstrafjustiz führten auch zu einer auf den ersten Blick geringfügigen, bei näherer Betrachtung jedoch nicht zu unterschätzenden Änderung des Strafprozeßrechts. Am 2. Oktober 1947 forderte Jakupow von der DJV die Ausarbeitung einer Anordnung, derzufolge Personen, „die wegen Nichterfüllung ihres Ablieferungssolls an landwirtschaftlichen Produkten

zum 1. 4. 1947 in Sachsen durchgeführten Schauprozessen um Wirtschaftsstrafverfahren; in Sachsen-Anhalt waren es neun von 21.

38 Die Schilderung des Falls bei Löwenthal, Geist, S. $176 \mathrm{f}$.; siehe auch den Vermerk über die Rücksprache von Jakupow mit Rosenthal-Pelldram am 3.4. 1947, BAB, DP1 VA Nr. 11, Bl. $110 \mathrm{f}$.

39 Vermerk über die Dienstbesprechung vom 16. 5. 1947, BAB, DP1 VA Nr. 7354.

$40 \mathrm{Vgl}$. die Aussage von Jakupow am 3. 4. 1947, in: Vermerk über die Rücksprache von Jakupow mit Rosenthal-Pelldram am 3. 4. 1947, BAB, DP1 VA Nr. 11, Bl. $110 \mathrm{f}$.

41 Vermerk Ackermanns, 11.9. 1947, BAB, DP1 VA Nr. 347, Bl. 34. Auch Arno Barth hegte große Vorbehalte gegen Schauprozesse: vgl. Weber, Justiz und Diktatur, S. 83. 
zu Freiheitsstrafen verurteilt worden" seien, verhaftet werden müßten, unabhängig davon, ob das Urteil in Kraft getreten sei oder nicht. Er berief sich dabei auf den angeblichen Unmut in der Bevölkerung darüber, daß wegen ihrer Verstöße gegen die Ablieferpflichten verurteilte Personen bis zum Inkrafttreten des Urteils sich oftmals noch mehrere Monate in Freiheit befänden. Der kommissarische Leiter der Abteilung Gesetzgebung Guski machte dagegen „prozeßrechtliche Bedenken“ geltend: „einerseits seien Strafurteile erst nach Inkrafttreten derselben zu vollstrecken, dem Erlaß eines Haftbefehls andererseits ständen aber meistenteils die Vorschriften des $₫ 112$ der Strafprozeßordnung entgegen“. Trotz dieser Einwände bestand Jakupow auf dem Erlaß einer entsprechenden Anordnung, deren Formulierung er der DJV überließ ${ }^{42}$.

Die DJV mußte somit auf sowjetischen Druck ${ }^{43}$ eine Verordnung erlassen, die von den rechtsstaatlichen Gepflogenheiten abwich. Da die Ausführung einer sowjetischen Anweisung nicht verweigert werden durfte, entschloß man sich in der DJV, die Verordnung auf jene Urteile zu beschränken, „durch die wegen vorsätzlicher Nichterfüllung des Ablieferungssolls an landwirtschaftlichen Erzeugnissen auf eine Freiheitsstrafe von mehr als zwei Monaten erkannt worden ist“. Außerdem enthielt $₫ 3$ des Verordnungsentwurfs den Hinweis, daß Haftentschädigung gezahlt werde, wenn im Rechtsmittelverfahren das direkt vollstreckte Urteil aufgehoben bzw. zugunsten des Angeklagten geändert worden sei ${ }^{44}$. Sowohl die einschränkende Klausel als auch $\ 3$ des Verordnungsentwurfs wurden auf Anordnung von Jakupow gestrichen; zusätzlich ordnete er an, „daß das in der Verordnung vorgesehene Verfahren auch auf Strafsachen Anwendung finden solle, in denen eine Bestrafung nach dem Kontrollratsgesetz Nr. 50 erfolge“ " ${ }^{45}$. Nachdem die DJV mit ihren Versuchen, die Auswirkungen der Verordnung zu begrenzen, gescheitert war, blieb ihr nichts anderes übrig, als in einem neuen Entwurf den Anordnungen der Rechtsabteilung Rechnung zu tragen. Nachdem dieser am 21. Oktober von Jakupow gebilligt worden war, trat die Verordnung durch Verkündung im Zentralverordnungsblatt umgehend in $\mathrm{Kraft}^{46}$. Am 23. Oktober 1947 versandte die DJV dazu eine erläuternde Rundverfügung, in der nicht nur darauf hingewiesen wurde, "daß schwere Verstöße gegen die in Betracht kommenden Strafbestimmungen auch wirklich mit schweren und der Verwerflichkeit der Taten entsprechenden Strafen geahndet werden" müßten, sondern auch die unbedingte Einhaltung der Verordnung angemahnt wurde. In diesem Zusammenhang erging außerdem die Anordnung, daß Richter oder Staatsanwälte, die dieser Ver-

42 Vermerk Walters über Besprechung mit Jakupow, 2. 10. 1947, BAB, DP1 VA Nr. 11, Bl. 124. \$112 StPO enthält die Voraussetzungen für die Verhängung der Untersuchungshaft.

43 Siehe zwei Vermerke Walters, 4. 10. 1947, über einen Anruf Jakupows in dieser Angelegenheit, BAB, DP1 VA Nr. 7181. Schönfeldt, Entwicklung, S. 146, führt „eine Intervention Eugen Schiffers" gegen den Erlaß der Verordnung an, kann dies jedoch nicht belegen.

44 Verordnungsentwurf, am 8.10.1947 der SMAD-Rechtsabteilung zur Genehmigung eingereicht, BAB, DP1 VA Nr. 7181.

45 Vermerk von Weiß über Unterredung vom 10.10. 1947, 15. 10. 1947, ebenda. Kontrollratsgesetz Nr. 50 vom 20.3. 1947 (Amtsblatt des Kontrollrats, Nr. 14, 31. 3. 1947, S. 266) betraf die Bestrafung der Entwendung und des rechtswidrigen Gebrauchs von zwangsbewirtschafteten Nahrungsmitteln und Gütern und von Urkunden, die sich auf Zwangsbewirtschaftung bezogen.

46 Vermerk über die Besprechung mit Jakupow, 21. 10. 1947, BAB, DP1 VA Nr. 7181; Verordnung über das Verfahren in Wirtschaftsstrafsachen, 21. 10. 1947, in: ZVOBl. 1947, S. 268. 
pflichtung nicht nachkämen, „wegen Verletzung ihrer Amtspflicht zur Verantwortung zu ziehen" seien ${ }^{47}$. Im Text der Rundverfügung hatte die SMAD-Rechtsabteilung bezeichnenderweise den Abschnitt gestrichen, in dem angemahnt wurde, bei geringfügigen Verstößen gegen die einschlägigen Strafbestimmungen keine Freiheitsstrafen zu verhängen, sondern sich in diesen Fälle mit Ordnungsstrafverfahren durch die Verwaltungsbehörden zu begnügen ${ }^{48}$.

Widerspruch gegen die Verordnung vom 21. Oktober regte sich in Thüringen, Sachsen-Anhalt und Brandenburg. Während in Weimar Bedenken gegen deren Durchführung mit dem alleinigen Gesetzgebungsrecht der Länder begründet und um eine Ausfertigung des sowjetischen Textes gebeten wurde ${ }^{49}$, brachte das Justizministerium in Halle sachliche Bedenken vor. Es verwies auf eine ähnliche, in einer Verordnung vom 13. März 1940 enthaltene Bestimmung, bei der es sich jedoch um eine „Kann-Bestimmung“ gehandelt habe ${ }^{50}$. Außerdem würden weite Bevölkerungskreise nicht verstehen, daß in Fällen, in denen mit der Haftvollstrekkung begonnen worden sei, in zweiter Instanz ein Freispruch erfolge. Wirtschaftssaboteure sollten zwar nicht auf freien Fuß gesetzt, aber in Untersuchungshaft gehalten werden ${ }^{51}$. Dies kam, wie die DJV in einem Antwortschreiben darlegte, jedoch nicht in Frage, da nach Verkündigung des Urteils nicht mehr Untersuchungshaft, sondern nur noch Strafhaft verhängt werden könne. Am Ende des auch den anderen Justizministerien zur Kenntnisnahme übersandten Schreibens mahnte die DJV „die durch die Verordnung vom 21. Oktober 1947 angeordnete sofortige Vollstreckung des Urteils“ an ${ }^{52}$.

In Potsdam lehnten sowohl der christdemokratische Justizminister Ernst Stargardt als auch Ministerialdirektor Hoeniger, Ministerialrat Horst Schulze, Generalstaatsanwalt Ostmann sowie Friedrich Ebert und Ministerpräsident Steinhoff von der SED die Verordnung ab. Auch sie sprachen der DJV das Recht ab, derartige Verordnungen zu erlassen; zudem befürchteten sie, daß es wegen der Verordnung „zwischen der Bauernbevölkerung einerseits und der SED, der Besatzungsmacht, der Justiz und dem neuen Staat andererseits zu einer starken Entfremdung kommen “ könne. Denn sie rechneten damit, daß viele erstinstanzliche Urteile bei Verstößen gegen die Ablieferpflichten „deshalb unrichtig und untragbar seien, weil sie auf einen Druck von Seiten [sic] des Kreiskommandanten oder einer örtlichen Parteistelle oder der Presse zustande" kämen und daher von der zweiten Instanz aufgehoben würden. Wenn es sich dadurch jedoch um ein bereits vollstrecktes Urteil handle, „so werde das Vertrauen zur Justiz und zum neuen Staat

47 DJV an Landesregierungen/Justizministerium, 23. 10. 1947, BAB, DP1 VA Nr. 7181.

48 Vermerk über die Besprechung mit Jakupow, 21. 10. 1947, ebenda.

+9 MdJ Thüringen an DJV, 10.11. 1947, ebenda. Über eine Reaktion auf dieses Schreiben ist nichts bekannt. Zum Konflikt mit Thüringen über die Gültigkeit von im Zentralverordnungsblatt abgedruckten Verordnungen siehe Kap. A.II.4.

50 Gemeint war die Verordnung zur Durchführung der Verordnung über die Zuständigkeit der Strafgerichte, die Sondergerichte und sonstige Strafverfahrensrechtliche Vorschriften, in: RGBI. 1940, I, S. 489.

51 MdJ Sachsen-Anhalt an DJV, 3. 12. 1947, BAB, DP1 VA Nr. 7187.

52 Chef der DJV an Landesregierung Sachsen-Anhalt/Justizministerium, 19. 2. 1948, BAB, DP1 SE Nr. 937. 


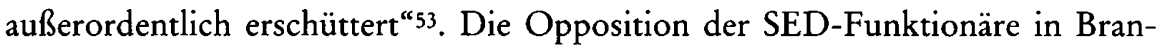
denburg resultierte somit nicht aus ihrem überzeugten Festhalten an rechtsstaatlichen Prinzipien, sondern aus einem pragmatisch-machtpolitischen Kalkül. Als Melsheimer und Benjamin am 6. November über Götz Berger von der ablehnenden Haltung Hoenigers erfuhren, waren sie „sehr empört" und betonten, daß die DJV sehr wohl zum Erlaß der Verordnung ermächtigt sei und daß auch justizpolitisch keine Bedenken bestünden. Auch die Abteilung Justiz beim SED-Zentralsekretariat machte sich diesen Standpunkt zu eigen. Hoeniger, der ebenfalls zu der Besprechung hinzustieß, blieb zwar bei seiner ablehnenden Stellungnahme, sah aber ein, daß eine weitere Opposition gegen die Verordnung zwecklos war ${ }^{54}$. Wie dieser Vorfall zeigt, folgte die SED nicht durchweg in justizpolitischen Fragen den Vorgaben der SMAD; sowjetische Anordnungen konnten auch parteiintern umstritten sein ${ }^{55}$. Letztlich hatte Widerstand gegen eine SMAD-Anordnung von solcher Bedeutung jedoch keine Chance.

Angesichts der grassierenden Kriminalität und der Notwendigkeit, die Wirtschaft wieder in Gang zu bringen, hielten alle Besatzungsmächte eine effektive Wirtschaftsstrafjustiz für erforderlich. Die sowjetische Besatzungsmacht glaubte freilich, Effektivität nur durch Einführung eigener Strafnormen und Prozeßformen sowie durch Außerkraftsetzung deutscher rechtsstaatlicher Grundsätze erreichen zu können. Dabei wurde die DJV zu einer reinen Befehlsübermittlungsstelle, die weder - wie bei Befehl Nr. 160 - selbständig Rechtsnormen auslegen, geschweige denn - wie bei der Verordnung über das Verfahren in Wirtschaftsstrafsachen - unter Verweis auf das Prozeßrecht Rechtsstaatlichkeit einfordern durfte. Auffällig bleibt jedoch, daß die SMAD-Rechtsabteilung Befehl Nr. 160 nur bei vorsätzlich, ja böswillig begangenen Sabotage- und Diversionsakten angewandt sehen wollte. Die Gerichte sollten die neue Strafnorm folglich nicht massenhaft, sondern, nicht zuletzt um der erzieherischen und abschreckenden Wirkung willen, gezielt und konsequent anwenden. Dies wurde freilich weder von den lokalen sowjetischen Kommandanten noch von den deutschen Justizorganen verstanden, so daß die Rechtsabteilung mit der Umsetzung des Befehls stets unzufrieden blieb.

\section{Die Einführung des Kassationsgesetzes}

Einen weiteren Eingriff der Besatzungsmacht in das deutsche Normensystem bildete 1946/47 die Einführung eines Kassationsgesetzes. Wenngleich die Aufhebung rechtskräftiger Urteile durch Kassation nach wie vor in den romanischen Ländern weit verbreitet ist und somit keineswegs als sowjetische Besonderheit gelten

53 Bericht, o.D., o.V. [vermutlich von Schäfermeyer], am 13.11. 1947 von Schäfermeyer an Ulbricht übersandt, SAPMO, NY 4182/1120, Bl. 122, $123 \mathrm{f}$.

54 Ebenda; Bericht Bergers über Besprechung mit Melsheimer und Benjamin, 8. 11. 1947, SAPMO DY 30 IV 2/13/407.

55 Wie aus dem Schreiben Schäfermeyers an Ulbricht vom 13.11. 1947 hervorgeht, waren auch die Zentralsekretariatsmitglieder nicht einer Meinung: Paul Merker, so Schäfermeyer, neige „zu dem Standpunkt Brandenburgs“, SAPMO, NY 4182/1120, Bl. 122. 
kann ${ }^{56}$, so war die Einführung dieses Rechtsinstituts in der SBZ eindeutig auf eine Anweisung der SMAD zurückzuführen, die dabei die strafprozessuale Praxis in ihrer Heimat vor Augen hatte. Am 16. September 1946 teilte Nikolajew Schiffer mit, daß die Oberlandesgerichtspräsidenten zwar von allen Verwaltungsaufgaben zu befreien seien ${ }^{57}$, aber nicht auf ihre Funktion als vorsitzende Richter der obersten Gerichte beschränkt werden dürften. „Sie müßten“, so fuhr er fort, „unbedingt das Recht haben, die rein gerichtliche Tätigkeit der zu ihrem Bezirk gehörenden Gerichte auf ihre Gesetzlichkeit nachzuprüfen und gegen ungerechte und ungesetzliche Urteile zu protestieren, gegen die das gesetzliche Rechtsmittel wegen der Rechtskraft des Urteils nicht mehr gegeben sei. [...] Das Ziel sei, derartige der materiellen Gerechtigkeit widersprechenden Urteile zu kassieren." In diesem Zusammenhang verwies er darauf, „daß in Rußland die Kassationsmöglichkeit gegeben sei mit der Maßgabe, daß sogar in geeigneten Fällen an Stelle der Kassation des Urteils die Herabsetzung der Strafe ausgesprochen werden könne“. Nikolajew war sich der Problematik dieses strafprozessualen Mittels angesichts der 1940 in das deutsche Recht eingeführten Nichtigkeitsbeschwerde und des außerordentlichen Einspruchs sehr wohl bewußt und bat daher um Schiffers Stellungnahme. Nach der anschließenden Beratung von Schiffer, Rosenthal-Pelldram und Nikolajew kam man jedoch zu dem Schluß, „daß diese außerordentliche Abänderungsmöglichkeit für rechtskräftige Urteile keinen Eingriff in die Unabhängigkeit der Richter bedeute". Schiffer sagte daher der Rechtsabteilung einen Verordnungsentwurf in dieser Angelegenheit $\mathrm{zu}^{58}$.

Die Zurückhaltung der sowjetischen Seite geht wohl nicht so sehr auf ihr rechtsstaatliches Bewußtsein, sondern auf ihr Bedürfnis zurück, sich gegenüber den Westalliierten als konsequente Gegnerin des „Faschismus“ zu präsentieren. Schiffer und seine nicht-kommunistischen Mitarbeiter sahen nach der Übertragung der Verwaltungsbefugnisse auf die Justizabteilungen in dem Kassationsrecht „ein gewisses Entgelt" für die Kompetenzeinbußen des Oberlandesgerichtspräsidenten ${ }^{59}$. Auch DJV-intern wurden der außerordentliche Einspruch und die Nichtigkeitsbeschwerde mit dem Ergebnis überprüft, „daß beide Institute wegen ihres nazistischen Charakters nicht mehr anwendbar seien, daß es vielmehr erforderlich sei, ein neues Verfahren, das den gegenwärtigen Verhältnissen entspreche, einzuführen" 60 . Der Wunsch der SMAD-Rechtsabteilung nach einem Verfahren zur Aufhebung rechtskräftiger Urteile stieß somit bei der DJV trotz der damit verbundenen Einschränkung der Rechtssicherheit nicht auf prinzipielle Ablehnung. Gegen die ursprüngliche sowjetische Absicht, die Kassation per SMAD-Befehl einzuführen, regte sich sowohl in der DJV als auch in der SED Widerspruch, da man dort die Setzung von deutschem Recht durch die Besatzungsmächte vermeiden wollte. Die SMAD stimmte auch dem ihr vorgeschlagenen Verfahren zu, demzufolge zunächst die DJV das Gesetz entwerfen und die am 20. Oktober ge-

56 Zur Verbreitung und zu den historischen Wurzeln der Kassation siehe Esch, Kassation, S. 8-16.

57 Siehe dazu Kap A.II.2.

58 Aktenvermerk über die Besprechung Schiffers mit Nikolajew am 19.9. 1946, BAB, DP1 VA Nr. 11, Bl. 69f.

59 So Winkelmann in: Vermerk über die Besprechung vom 16. 10. 1946, BAB, DP1 VA Nr. 7354.

60 So Weiß in: Protokoll über die Länderkonferenz vom 1./2. 11. 1946, BAB, DP1 VA Nr. 22, Bl. 67. 
wählten Landtage es beraten und verabschieden sollten ${ }^{61}$. Dies konnte der Besatzungsmacht nur recht sein, ermöglichte es ihr doch, sich bei der Einführung dieses aus dem sowjetischen Justizwesen stammenden Rechtsinstituts im Hintergrund zu halten.

Der am 27. September 1946 eingereichte erste Gesetzesentwurf stieß jedoch nicht auf Zustimmung bei der SMAD-Rechtsabteilung. Nikolajew bestand - im Gegensatz zu den weiter gefaßten Bestimmungen des DJV-Entwurfs - darauf, eine Kassation nur dann zuzulassen, „wenn gesetzliche Revisionsgründe vorlägen“. Außerdem hielt er einen neu zu errichtenden Kassationshof bei den Oberlandesgerichten für überflüssig und erklärte die Strafsenate für zuständig. Auf seine Anordnung62 wurde der Länderkonferenz am 1./2. November 1946 ein nach sowjetischen Vorgaben umgearbeiteter Gesetzesentwurf vorgelegg ${ }^{63}$. Nach den Darlegungen von Wolfgang Weiß sollte sich die (damals noch so genannte) „außerordentliche Revision in Strafsachen" nur gegen ein rechtskräftiges Urteil richten, wenn dieses „auf einer Gesetzesverletzung im Sinne der Revisionsvorschriften der Strafprozeßordnung beruhe". Antragsberechtigt sei der Oberlandesgerichtspräsident; das Verfahren solle vor einem Senat des Oberlandesgerichts nach den Vorschriften der Strafprozeßordnung über das Revisionsverfahren geführt werden. Wie so oft, benutzte die SMAD-Rechtsabteilung die Länderkonferenz, um durch autoritative Äußerungen das "Gesetzgebungsverfahren“ voranzubringen. Jakupow begründete die Notwendigkeit eines Kassationsgesetzes mit den bei Revisionen häufig festgestellten großen Fehlern in der Urteilsfindung. Dabei trat er der Auffassung von Wolfgang Lange (Schwerin) entgegen, der die richterliche Unabhängigkeit des Oberlandesgerichtspräsidenten als gefährdet ansah, wenn ihm die Entscheidung über die Einleitung eines Kassationsverfahrens überlassen würde. Richterliche Unabhängigkeit bedeutete demgegenüber für Jakupow, „daß der Richter sich bei der Entscheidung eines konkreten Falles nach den geltenden Gesetzen zu richten habe und daß hierbei niemandem das Recht gegeben sei, den Richter zu veranlassen, anders zu entscheiden, als es seiner Überzeugung entspreche“. „Unabhängigkeit des Richters", so fuhr er fort, „heiße aber nicht, daß seine Tätigkeit jeder Kontrolle entzogen sei. Auch die Richter machten Fehler, und diese Fehler müßten beseitigt werden." Wie er bereits einige Tage zuvor Melsheimer mitgeteilt hatte ${ }^{64}$, wollte er den Entwurf der DJV dadurch ergänzen, daß auch dem Generalstaatsanwalt die Entscheidung über die Einleitung eines Kassationsverfahrens übertragen werde, und zudem eine Kassation auch dann ermöglichen, wenn „die anerkannte Strafe zu schwer oder zu gering sei, oder [...] das Urteil aus

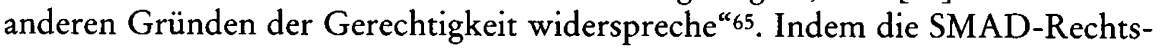

61 So Nathan in: Stenographische Niederschrift über die Sitzung des Ausschusses für Rechtsfragen am 21./ 22. 6. 1947, SAPMO, DY 30 IV 2/1.01/51, Bl. $33 \mathrm{f}$.

62 Aktenvermerk über von Stackelbergs Rücksprache mit Nikolajew am 3. 10. 1946, BAB, DP1 VA Nr. 11, Bl. 76. Der DJV-Entwurf lag dem Vf. nicht vor.

63 DJV an SMAD-Rechtsabteilung, 21. 10. 1946, BAB, DP1 VA Nr. 6957, Bl. 99. Die Darstellung von Amos, Justizverwaltung, S. $194 \mathrm{f}$., die DJV sei von der SMAD-Rechtsabteilung im Januar/Februar 1947 mit der Ausarbeitung eines Kassationsgesetzentwurfs beauftragt worden, der dann im April 1947 auf einer Länderkonferenz beraten worden sei, ist unzutreffend.

64 Aktenvermerk über die Besprechung von Melsheimer mit Jakupow und Schitomirsky am 29.10. 1946, BAB, DP1 VA Nr. 11, Bl. 80.

65 Protokoll der Länderkonferenz vom 1./2. 11. 1946, BAB, DP1 VA Nr. 22, Bl. 66f., 69-71. 
abteilung die Kassation nicht nur bei einer formellen, sondern auch bei einer materiellen Rechtsverletzung zulassen wollte, wich sie, aus unbekannten Gründen, von ihrer bisherigen restriktiven Praxis ab. Mit der Erweiterung der Kassationsvoraussetzungen nahm sie jedoch Rechtskraftdurchbrechungen in Kauf, die notwendigerweise mit einem Verlust an Rechtsstaatlichkeit verbunden waren.

Aufgrund dieser Anweisung legte die DJV einen neuen Gesetzesentwurf vor, den die SMAD-Rechtsabteilung nochmals abänderte und daraufhin anordnete, ihn den Landesparlamenten zur Zustimmung zu übersenden ${ }^{66}$. Nun wurde auch die SED-Führung in das „Gesetzgebungsverfahren “ einbezogen. Nachdem Polak nach eigener Darstellung eher zufällig in Karlshorst von dem Entwurf erfahren hatte, wurde die SED-Justizabteilung von der SMAD-Rechtsabteilung genauer informiert und billigte das Vorhaben ${ }^{67}$. Am 28. Februar 1947 übermittelte Melsheimer Polak den endgültigen Entwurf mit der Bitte, ihn den SED-Landtagsfraktionen mit der Maßgabe zu übersenden, keinen Widerspruch dagegen zu erheben. Ähnliche Schreiben wollte die DJV an die anderen Blockparteien richten ${ }^{68}$. Am 8. März übersandte Schiffer den Gesetzesentwurf den Justizministerien. Sein Begleitschreiben schloß mit den mahnenden Worten: „Da der beigefügte Entwurf die Billigung der Rechtsabteilung der SMAD gefunden hat und diese den auch von der Deutschen Justizverwaltung dringend befürworteten Wunsch hat, daß diese Frage in den Ländern und Provinzen der Zone nach Möglichkeit einheitlich geregelt wird, würde es begrüßt werden, wenn der Gesetzentwurf in der hiermit vorgelegten Form zum Gesetz erhoben würde."69 Seit der Intervention der SMAD-Rechtsabteilung von Ende Oktober/Anfang November waren anscheinend keine wesentlichen Änderungen mehr vorgenommen worden. Denn der Entwurf sprach das Antragsrecht sowohl dem Oberlandesgerichtspräsidenten als auch dem Generalstaatsanwalt zu und legte fest, daß ein Kassationsverfahren nur eingeleitet werden könne, wenn das Urteil auf einer Gesetzesverletzung im Sinne der Revisionsvorschriften der Strafprozeßordnung beruhe oder wenn es "wegen eines offenbaren Fehlers bei der Strafbemessung gröblich der Gerechtigkeit" widerspreche. Ein Kassationsantrag konnte innerhalb einer Frist von einem Jahr nach Eintreten der Rechtskraft gestellt werden ${ }^{70}$.

Trotz der Ermahnungen Schiffers und der SED-Parteiführung verabschiedete nur der Landtag von Sachsen-Anhalt ein Kassationsgesetz, das jedoch die Antragsfrist auf sechs Monate herabsetzte und die zweite Kassationsvoraussetzung durch die Streichung der Worte „wegen eines offenbaren Fehlers der Strafbemessung “ änderte ${ }^{71}$. In den anderen Ländern scheiterte der Entwurf aus den unterschiedlichsten Gründen. Im Justizausschuß des Weimarer Landtages widersetzten

66 Bericht Nathans in: Stenographische Niederschrift über die Sitzung des Ausschusses für Rechtsfragen am 21./22. 6. 1947, SAPMO, DY 30 IV 2/1.01/51, Bl. 34. Vgl. auch Amos, Justizverwaltung, S. 196.

67 So Polak in: Stenographische Niederschrift über die Sitzung des Ausschusses für Rechtsfragen am 21./ 22. 6. 1947, SAPMO, DY 30 IV 2/1.01/51, Bl. $47 \mathrm{f}$.

68 Melsheimer an Polak, 28.2. [1947], SAPMO, NY 4182/1120, Bl. 15.

69 Chef der DJV an Landes- und Provinzialregierungen/Justizministerium, 8. 3. 1947, BAB, DP1 VA Nr. 6596, Bl. 156.

70 Entwurf eines Gesetzes über die Kassation rechtskräftiger Urteile in Strafsachen, ebenda, Bl. 157.

7 Gesetz über die Kassation rechtskräftiger Urteile in Strafsachen, in: Gesetzblatt der Provinz Sachsen-Anhalt I, 1947, S. 84. 
sich die CDU- und LDP-Abgeordneten insbesondere mit dem Argument, daß das Gesetz einen Eingriff in die Unabhängigkeit der Gerichte darstelle. In Schwerin war der Entwurf nach Auskunft von Hans Sieber (SED) bis Mitte Juni 1947 im Rechtsausschuß noch nicht behandelt worden. Jedoch wollten der SED-Landesvorstand und der Ministerrat das Antragsrecht nicht dem Oberlandesgerichtspräsidenten, sondern der Volksvertretung zusprechen; außerdem sollte ein besonderer, auch mit Laien zu besetzender Kassationsgerichtshof gebildet werden. Die Rechtsausschüsse der Landtage in Brandenburg und Sachsen wiederum wollten die umfangreichen Möglichkeiten aufrechterhalten, die die dort noch angewandte Nichtigkeitsbeschwerde ${ }^{72}$ gegenüber dem Kassationsgesetz bot. So bemängelte der Rechtsausschuß in Potsdam, daß eine Nachprüfung der Beweiswürdigung nach dem Kassationsgesetz nicht mehr möglich sei und daß dieses sich nur auf Urteile, nicht aber, wie die Nichtigkeitsbeschwerde, auch auf Beschlüsse beziehe. Fritz Geyer (SED), der als Staatssekretär das Büro des sächsischen Ministerpräsidenten leitete ${ }^{73}$, sprach sich aus taktischen Gründen für das Kassationsgesetz aus: Zwar handle es sich bei der Nichtigkeitsbeschwerde nicht um ein „ausgesprochen nazistisches Rechtsgut“, aber sie stehe „mit der ganzen Nazigesetzgebung in $\mathrm{Zu}$ sammenhang“, so daß es besser sei, „solche Gesetze durch Neuformulierungen zu ersetzen“. Der Rechtsausschuß des sächsischen Landtags sah vor diesem Hintergrund die Kassationsvoraussetzungen des Gesetzesentwurfs als zu eingeschränkt an: „gerade die Urteile, für die wir die Kassation brauchen, nämlich die politisch untragbaren", könne man damit nicht erfassen. Daher schlug er vor, die Worte „bei der Strafbemessung“ zu streichen. Außerdem sollte nicht der Oberlandesgerichtspräsident, sondern der Generalstaatsanwalt antragsberechtigt sein. Dabei war für Geyer nicht maßgebend, daß dann derjenige, der den Kassationsantrag stelle, möglicherweise auch darüber entscheide, sondern daß der Oberlandesgerichtspräsident politisch unabhängig, der Generalstaatsanwalt jedoch politisch abhängig sei und den Weisungen der Justizverwaltung und der Regierung unterliege ${ }^{74}$. Es war somit keine Einheitsfront von Kämpfern für den Rechtsstaat, die die Einführung des Kassationsgesetzes im ersten Anlauf verhinderte, sondern der von der Besatzungsmacht wieder ins Leben gerufene deutsche Föderalismus, der sich damals noch nicht, wie dies der SMAD-Rechtsabteilung, der DJV und der SED-Führung vorschwebte, im Sinne der Zentralinstanzen instrumentalisieren ließ.

Es spricht für die wachsende Bedeutung der SED in der Justizpolitik, daß nun der SED-Zentrale in Berlin eine koordinierende Funktion zukam. Am 21./ 22. Juni 1947 befaßte sich der SED-Ausschuß für Rechtsfragen mit dem Gesetzes-

72 Zu Sachsen: siehe Urteil des OLG Dresden vom 18. 12. 1946, in: NJW 1 (1947/48), S. 396. Zu Brandenburg siehe die Äußerung von Horst Schulze in: Stenographische Niederschrift über die Sitzung des Ausschusses für Rechtsfragen am 21./ 22.6. 1947, SAPMO, DY 30 IV 2/1.01/51, Bl. 38. In den Westzonen durfte sie nicht mehr angewandt werden, was in Juristenkreisen teilweise bedauert wurde: vgl. Full, Nichtigkeitsbeschwerde.

73 Vgl. zur Person Richter/Schmeitzner, Einer von beiden, S. 25.

74 Zur Behandlung des Kassationsgesetzentwurfs in den Landtagen siehe den Bericht Nathans sowie die Äußerungen von Schulze, Geyer, Schultes und Sieber in: Stenographische Niederschrift über die Sitzung des Ausschusses für Rechtsfragen am 21./ 22.6. 1947, SAPMO, DY 30 IV 2/1.01/51, Bl. 34f., 37-47. 
entwurf. Während Nathan von der DJV über den Stand der Angelegenheit berichtete, referierten die Repräsentanten aus den Ländern über die Behandlung der Gesetze in den Landesparlamenten. Im Unterschied zu den meisten SED-Juristen aus den Ländern trat Polak für den Gesetzesentwurf ein. Den Darlegungen der SMAD-Rechtsabteilung und der DJV folgend, bezeichnete er die Nichtigkeitsbeschwerde als „ein nazistisches Gesetz“, das nicht mehr angewandt werden dürfe. Die Differenzen zwischen beiden strafprozessualen Mitteln seien zudem nicht gradueller, sondern prinzipieller Natur, da bei der Kassation „das Institut der Rechtskraft “ seine Wirksamkeit behalte. Zudem verteidigte er das Antragsrecht des Oberlandesgerichtspräsidenten, der nach den Landesverfassungen eben kein unabhängiger Richter, sondern vom Landtag gewählt und diesem auch für die Rechtsprechung in seinem Gebiet verantwortlich sei. Umstritten war auch die Vorgehensweise bei der Einführung des Kassationsgesetzes. Nathan schilderte eindringlich die Zwangslage der DJV, die gleichzeitig mit SMAD-Vorgaben und den damit nicht zu vereinbarenden Änderungswünschen der Länder konfrontiert würde. Auch wenn der Entwurf Mängel aufweise, dürfe man nicht vergessen: „Wir haben nicht freie Hand, wir sind hier wirklich nur ausführendes Organ der SMA, die wünscht, daß der Entwurf in dieser Form Gesetz wird. " Geyer wollte das nicht hinnehmen und forderte die DJV auf, die Beanstandungen aus den Landtagen der SMAD-Rechtsabteilung mit der Empfehlung zu übermitteln, sich "dieser Kritik zu fügen“. Bei dem derzeitigen Entwicklungsstadium könne auch die SED „unserem Landtage nicht einfach sagen: Ihr müßt das schlucken, und wenn es vom deutschen Standpunkt aus noch so verkehrt ist." Notfalls solle die SMAD eben einen Befehl herausgeben. Geyer, weder ein überzeugter Föderalist noch ein Anhänger der Parlamentarismus, wollte damit zum einen über den Landtag den Einfluß der SED auf die Formulierung des Kassationsgesetzes sichern und zum anderen aus Gründen der Außenwirkung eine Instrumentalisierung der Landtage im Sinne von SMAD und DJV vermeiden. Angesichts der Uneinigkeit in der Sache und in der Vorgehensweise schlugen die Vertreter des zentralen SED-Apparats eine vermittelnde Linie ein. Schäfermeyer und Fechner sprachen sich dafür aus, den Gesetzesentwurf dem Zentralsekretariat vorzulegen; anschließend, so Fechner, wolle man gegebenenfalls die SMAD zum Einlenken bewegen ${ }^{75}$.

Am 15. August stimmte das Zentralsekretariat einer von Fechner und Ulbricht unterzeichneten Vorlage zu, die den gegenüber März 1947 unveränderten Gesetzentwurf enthielt. In einem erläuternden Zusatz hieß es: „Dieser Gesetzentwurf ist durch die Zentralverwaltung für Justiz mit den Ländern vereinbart. Unterschiedlich ist die Auffassung in den einzelnen Ländern über die Frage, wer die Kassation beantragen kann. Der rechtspolitische Ausschuß wie auch die Abteilung Justiz beim Zentralsekretariat entschied [sic] sich in Übereinstimmung mit den Genossen von der Zentralverwaltung für Justiz für die im $\$ 1$ gefundene Formulierung. " ${ }^{66}$ Die mangelnde Berücksichtigung der Bedenken aus den Ländern wurde

75 Die Äußerungen von Nathan, Polak, Geyer, Schäfermeyer und Fechner ebenda, Bl. 36, 48f., 42, 52, 54, 56. Zur Diskussion vgl. auch Amos, Justizverwaltung, S. 195-197.

76 Protokoll der Zentralsekretariatssitzung am 15. 8. 1947, SAPMO, DY 30 IV 2/2.1/117, TOP 14, Anlage 2 (dort auch das Zitat). Nach $\$ 1$ stand dem Generalstaatsanwalt und dem Oberlandesge- 
dabei ebenso verschwiegen wie die Tatsache, daß die SMAD-Rechtsabteilung den Entwurf bereits seit längerem bestätigt hatte. Ungeachtet der vollmundigen Aussage Fechners, derzufolge die Freundschaft zwischen SMAD und SED nicht einseitig sein dürfe ${ }^{77}$, hatte das Zentralsekretariat die von Karlshorst genehmigte Fassung zu ihrer eigenen gemacht. In einem zweiten Anlauf verabschiedeten im September und Oktober 1947 die Landtage in Brandenburg, Sachsen, Mecklenburg und Thüringen nach einem erneuten, in anderem Zusammenhang vorgenommenen Appell des SED-Zentralsekretariats ${ }^{78}$ und auf Druck der sowjetischen Militärverwaltung das Kassationsgesetz in der bestätigten Fassung ${ }^{79}$.

Die Einführung der Kassation in der SBZ ging ebenso auf eine sowjetische Anordnung zurück ${ }^{80}$ wie die Erweiterung der Kassationsvoraussetzungen, die nicht nur, wie die DJV es anstrebte, bei einer formellen, sondern auch bei einer materiellen Rechtsverletzung gegeben sein sollten. Die Überlegung, das Gesetz zentral auszuarbeiten, aber durch die Landtage verabschieden zu lassen, hatte indes in der DJV und in der SED-Führung ihren Ursprung. Jedoch erkannte auch die SMADRechtsabteilung die für sie damit verbundenen Vorteile: Während sie sich im Hintergrund hielt, konnte ein den deutschen rechtsstaatlichen Traditionen widersprechendes Gesetz auf diese Weise demokratisch legitimiert werden. Die Landtage erwiesen sich dabei als Bremser - wenngleich die dort geäußerten Bedenken keineswegs nur die mangelnde Rechtsstaatlichkeit des Gesetzesentwurfs, sondern auch die gegenüber der Nichtigkeitsbeschwerde geringeren Eingriffsmöglichkeiten betrafen. Nachdem die DJV sich gegenüber den Landtagen nicht hatte durchsetzen können, versuchte die SED-Zentrale die Koordination zu übernehmen. Auch innerhalb der Partei prallten die unterschiedlichen Meinungen aufeinander; der für alle Parteigliederungen verbindliche Zentralsekretariatsbeschluß ging über diese Differenzen hinweg und vermied einen Konflikt mit der SMAD-Rechtsabteilung, indem er sich deren Position zu eigen machte. Mit der Herstellung dieses Gleichklangs zwischen SMAD-Rechtsabteilung und SED-Führung war eine wesentliche Hürde auf dem Weg zur Verabschiedung des Gesetzes in den Landtagen beseitigt, wenngleich auch in dieser Phase des "Gesetzgebungsverfahrens" die Einheitspartei auf sowjetische „Unterstützung“ angewiesen war.

richtspräsidenten das Antragsrecht zu. Vgl. Amos, Justizverwaltung, S. $197 \mathrm{f}$., die jedoch den Eindruck vermittelt, als wäre der Entwurf von den SED-Gremien noch verändert worden.

77 Stenographische Niederschrift über die Sitzung des Ausschusses für Rechtsfragen am 21./22.6. 1947, SAPMO, DY 30 IV 2/1.01/51, Bl. 56.

78 Rundschreiben des SED-Zentralsekretariats, 10. 9. 1947, Betr.: Durchführung des Befehls Nr. 201 über die Entnazifizierung in der sowjetischen Besatzungszone, in: Rößler, Entnazifizierungspolitik, S. 171.

79 Die Kassationsgesetze (in Brandenburg hieß es nach wie vor „Gesetz über die Nichtigkeitsbeschwerde gegen Strafurteile") in: Gesetz- und Verordnungsblatt der Landesregierung Brandenburg 1947, S. 23 (11. 9. 1947); Gesetze, Befehle, Verordnungen, Bekanntmachungen veröffentlicht durch die Landesregierung Sachsen, 1947, S. 445 (3. 10. 1947); Regierungsblatt für Mecklenburg 1947, S. 255 f. (18. 9. 1947); Regierungsblatt für das Land Thüringen I, 1947, S. 81 (10. 10. 1947). Das brandenburgische Gesetz wich insofern leicht von der Vorlage ab, als es das Antragsrecht nur dem Generalstaatsanwalt zugestand.

80 Dies wird in der Darstellung von Benjamin u.a., Zur Geschichte der Rechtspflege 1945-1949, S. 283, verschleiert. 


\section{Die Wiedereinführung und Einschränkung der Verwaltungsgerichtsbarkeit}

„Die Verwaltungsgerichtsbarkeit ist der Prüfstein des Rechtsstaats."81 Diesen Grundsatz hatten offensichtlich auch die Alliierten vor Augen, als sie mit Kontrollratsgesetz Nr. 36 am 10. Oktober 1946 die Wiedererrichtung von Verwaltungsgerichten dekretierten ${ }^{82}$. Es handelte sich dabei freilich um ein bloßes Rahmengesetz, das die Zonenbefehlshaber ermächtigte, Ausführungsbestimmungen über Organisation und Zuständigkeit der Verwaltungsgerichte zu erlassen. Die sowjetische Besatzungsmacht stand diesem Zweig der Gerichtsbarkeit distanziert gegenüber. Denn auf die Ankündigung Schiffers vom 30. Oktober 1945, daß er unter anderem „die Fragen der Verwaltungsgerichtsbarkeit" überprüfen werde, antwortete Karassjow am 14. November: „Insbesondere beachten Sie, inwieweit die Wiedererrichtung von Verwaltungsgerichten wirklich zweckmäßig erscheint, und ob dies nicht zu einer Abschwächung der Verantwortung der Beamten gegenüber den von ihnen begangenen Rechtsverstößen führt." 83

In einer daraufhin ausgearbeiteten, am 10. Dezember 1945 in Karlshorst eingereichten Denkschrift sprach sich Schiffer - wie bereits in seinem Werk „Die Deutsche Justiz"84 - für die Aufhebung einer von der ordentlichen Gerichtsbarkeit getrennten Verwaltungsgerichtsbarkeit aus. Diese Trennung sei im 19. Jahrhundert aus personellen Erwägungen vorgenommen worden; die bei den Verwaltungsgerichten eingesetzten Ziviljuristen hätten sich jedoch „in der Praxis hervorragend bewährt". Außerdem hätten sich das Privatrecht und das öffentliche Recht mehr und mehr angenähert, so daß er dafür plädierte, „innerhalb der ordentlichen $\mathrm{Ge}$ richte besondere Abteilungen (Kammern, Senate) für verwaltungsrechtliche Streitigkeiten“ zu errichten. Die Zuständigkeit der Verwaltungsgerichtsbarkeit wollte er nicht durch das Enumerationsprinzip begründen, sondern durch eine weit gefaßte Generalklausel ${ }^{85}$, „die nur ausnahmsweise durch Gesetz eingeengt werden könnte" 86 . Während sich die Rechtsabteilung zur Zuständigkeit nicht äußerte, betonte Karassjow wiederholt gegenüber Schiffer, daß er mit seiner Auffassung übereinstimme, derzufolge die Verwaltungsgerichtsbarkeit den ordentlichen $\mathrm{Ge}-$ richten zu übertragen sei ${ }^{87}$. Schiffers Denkschrift wurde daher bei den Verhand-

81 Stolleis, Verwaltungsgerichtsbarkeit, S. 26.

82 Gedruckt in: Amtsblatt des Kontrollrats, Nr. 11, 31. 10. 1946, S. 183.

83 Chef der DJV an Chef der SMAD-Rechtsabteilung, 30.10. 1945, Chef der SMAD-Rechtsabteilung an Chef der DJV, 14. 11. 1945, BAB, DP1 VA Nr. 2, Bl. 96, 103.

${ }_{84}$ Schiffer, Deutsche Justiz, 1. Aufl., S. 281-284; 2. Aufl., S. 210-216.

85 Nach dem Enumerationsprinzip können nur bestimmte, im Gesetz aufgeführte Verwaltungsakte, nach der Generalklausel können alle behördlichen Verfügungen, sofern sie die davon Betroffenen in ihren Rechten verletzen, gerichtlich angefochten werden.

86 Eugen Schiffer, Frage der Wiedereinrichtung von Verwaltungsgerichten, 5. 12. 1945, SAPMO NY 4174/5, Bl. 134-136 (mit dem Vermerk: „eingereicht in Karlshorst am 10/12.45“). Die Denkschrift auch in: BAB, DP1 VA Nr. 6928 (so Heil, Verwaltungsgerichtsbarkeit, S. 68). Die Akte konnte vom Vf. trotz mehrmaliger Bestellung im Bundesarchiv nicht eingesehen werden; sie scheint verlorengegangen zu sein.

87 Aktenvermerk über die Besprechung Schiffers mit Karassjow am 18. 3. 1946; Aktenvermerk über die Rücksprache Schiffers mit Karassjow am 17.5. 1946, BAB, DP1 VA Nr. 11, Bl. 28, 40. Heil, Verwaltungsgerichtsbarkeit, S.68, geht hingegen davon aus, daß dic SMAD den Wiederaufbau einer gesonderten Verwaltungsgerichtsbarkeit angestrebt habe. 
lungen über die Verwaltungsgerichtsbarkeit im Alliierten Kontrollrat von der sowjetischen Seite zur Stützung ihrer Position eingesetz ${ }^{88}$; im März 1946 teilte Karassjow Schiffer jedoch mit, daß sich die westlichen Alliierten gegen die von ihm favorisierte Regelung aussprächen ${ }^{89}$. Letztlich setzten diese sich durch, und die Sowjetunion, die auch hier die Einheit der Siegermächte nicht gefährden wollte, trug die mit Kontrollratsgesetz Nr. 36 verkündete Entscheidung zur Wiedererrichtung der Verwaltungsgerichtsbarkeit mit.

Um dieses Rahmengesetz für die eigene Zone auszufüllen, beauftragte die SMAD-Rechtsabteilung Anfang November 1946 die DJV damit, zwei unterschiedliche Vorschläge einzureichen ${ }^{90}$. Im Zuge dieser Arbeiten wurde auf Initiative der DJV am 28. November 1946 eine Besprechung mit den Spezialisten auf diesem Gebiet aus den Ländern einberufen"1, wo Schiffer und Kleikamp mit Nachdruck für die Generalklausel eintraten. Thüringen verfügte als einziges Land der SBZ seit Juni 1946 über ein Oberverwaltungsgericht, das bereits vor der Konferenz gegenüber der DJV die Generalklausel als „das Kernstück des Rechtsstaates und aller Verwaltungsgerichtsbarkeit" bezeichnet hatte ${ }^{22}$. Alle anderen Länder sprachen sich jedoch gegen die Generalklausel und für eine mindestens vorübergehende Zuständigkeitsregelung nach dem Enumerationsprinzip aus, da sie Beeinträchtigungen ihrer Verwaltungstätigkeit befürchteten. Brandenburg wollte darüber hinaus eine Kontrolle der Verwaltungsgerichtsbarkeit durch die Landtage, um „reaktionären Tendenzen“ der Verwaltungsrechtsprechung entgegenwirken zu können'3. Ungeachtet ihrer mangelnden Unterstützung durch die Länder, befürworteten die von der DJV bei der SMAD-Rechtsabteilung eingereichten Verordnungsentwürfe eine „nahezu unbeschränkte[.] Generalklausel“ ${ }^{\text {“94. }}$.

Als die SED-Justizabteilung von den Vorbereitungen zur Einführung der Verwaltungsgerichtsbarkeit erfuhr, schaltete sie sich umgehend in die Diskussion ein 95 . Wahrscheinlich ist die Initiative zur Stellungnahme vom 26. November Polak zuzuschreiben, der sich in der damaligen Verfassungsdiskussion nachdrücklich gegen eine Gewaltenteilung aussprach\%6. Die SED-Justizabteilung sah in der Verwaltungsgerichtsbarkeit einen Störfaktor beim Aufbau einer neuen Staats- und Gesellschaftsordnung. Dennoch sah sie sich aus vier Gründen nicht in der Lage, der Errichtung von Verwaltungsgerichten eine Absage zu erteilen. Er-

88 Vgl. den Aktenvermerk Schiffers, 8. 1. 1946, BAB, DP1 VA Nr. 11, Bl. 4f., demzufolge Nikolajew ihm mitgeteilt hatte, daß seine Denkschrift über die Verwaltungsgerichtsbarkeit beim Kontrollrat liege.

89 Aktenvermerk über die Besprechung Schiffers mit Karassjow am 18. 3. 1946, ebenda, Bl. 40.

90 Vgl. Heil, Verwaltungsgerichtsbarkeit, S. 66, Anm. 16.

91 Siehe Aktenvermerk über die Besprechung Schiffers mit Karassjow am 13.11. 1946, BAB, DP1 VA Nr. 11, Bl. 81; vgl. Loening, Kampf, S. 77.

92 Ebenda, S. 76.

93 Zum Verlauf der Konferenz Heil, Verwaltungsgerichtsbarkeit, S. $68 \mathrm{f}$.

94 Ebenda, S. 68. Meyer, Entwicklung, S. 562, schreibt - wohl aus eigener Erinnerung -, daß die Entwürfe um die Jahreswende $1946 / 47$ in der DJV entstanden seien. Meyer war damals Vortragender Rat in der damit befaßten Abteilung V.

95 Heil, Verwaltungsgerichtsbarkeit, S. 169, meint unzutreffend, daß sich bei der SED erst „im März/ April 1947 die zukünftige Linie in der bis dahin unentschiedenen Zuständigkeitsfrage “ deutlich abgezeichnet habe.

96 Siehe Polak, Wesen; Abteilung Justiz, Stellungnahme zu den Verwaltungsgerichten, 26. 11. 1946, SAPMO, NY 4182/1119, Bl. 50-61. Vgl. dazu auch Amos, Justizverwaltung, S. 189 f. 
stens existierte ein entsprechendes Kontrollratsgesetz, auf dessen Durchführung die SMAD bestand. Zweitens wollte sich die Justizabteilung nicht dem Vorwurf aussetzen, „keine Rechtsgarantien gegenüber der Verwaltung zu schaffen“. Drittens enthielt der SED-Verfassungsentwurf einen Artikel, demzufolge die Verwaltungsgerichtsbarkeit dem „Schutze der Bürger gegen widerrechtliche Anordnungen und Verfügungen der Verwaltungen" diene ${ }^{97}$, und viertens schien es allemal besser, besondere Verwaltungsgerichte einzurichten, als den ordentlichen Gerichten die Kontrolle über die Verwaltung zu überlassen. Als Lösung des Problems das auch innerhalb der SED kontrovers diskutiert wurde ${ }^{98}$ - schlug die Justizabteilung vor, die Verwaltungsgerichte in ihrer Unabhängigkeit und Wirksamkeit erheblich zu beschneiden, indem nur ein Verwaltungsgericht pro Land eingerichtet wurde, dessen Richter für eine bestimmte Frist vom Parlament zu wählen waren. Zudem trat sie für das Enumerationsprinzip und dafür ein, bestimmte legislative Akte von vornherein zu „Regierungsakten“ zu erklären, die der Überprüfung durch die Verwaltungsgerichtsbarkeit entzogen waren. Um ihr Vorhaben zu realisieren, ging die SED-Zentrale zweigleisig vor. Am 20. Dezember 1946 brachte Polak in einer Beratung mit dem DVdI-Präsidenten Erich Reschke und den Innenministern von Sachsen, Mecklenburg, Brandenburg und der Provinz Sachsen die Problematik und das Ziel der SED auf die knappe Formel: „Es müssen Verwaltungsgerichte als besondere Gerichte geschaffen werden. Es ist der Plan, diese Gerichte möglichst ungefährlich zu machen." Dazu sollte das SED-Zentralsekretariat einen entsprechenden Gesetzesentwurf den Landesvorständen zustellen, der gegebenenfalls in den Landtagen eingebracht werden konnte 99 . Parallel dazu unterbreitete das Zentralsekretariat den Gesetzesentwurf der SMAD-Rechtsabteilung ${ }^{100}$, bei der somit die Fäden in dieser Frage zusammenliefen.

Aufgrund der Vorlagen von SED und DJV erstellte die SMAD-Rechtsabteilung einen eigenen Entwurf, den sie nach einer entsprechenden Ankündigung 101 im April 1947 der DJV zukommen ließ. Sie hatte fast ausschließlich die SED-Vorschläge aufgegriffen. Dies galt vor allem für die Zuständigkeitsregelung: Statt der von der DJV empfohlenen Generalklausel sollte, in den Worten Schiffers, „das Enumerationsprinzip in schroffster Form gelten“. Da dieser keine Möglichkeit mehr sah, die DJV-Vorschläge zu realisieren, schlug er der SMAD-Rechtsabteilung am 13. Mai vor, „die Wiedereinrichtung der Verwaltungsgerichte ,bis zu ei-

${ }^{97}$ Siehe Art. 95 des Entwurfs einer Verfassung für die Deutsche Demokratische Republik, verabschiedet vom SED-Parteivorstand am 14. 11. 1946, in: Dokumente der SED, Bd. 1, S. 134.

98 Einige sächsische SED-Genossen traten für eine Verwaltungsgerichtsbarkeit „nur durch besondere Parlamentsausschüsse“ ein; außerdem sprachen sie sich für das Enumerationsprinzip aus, während die Justizabteilung der thüringischen SED eine eingeschränkte Generalkausel befürwortete. Siche Abteilung Justiz, Stellungnahme zu den Verwaltungsgerichten, 26. 11. 1946, SAPMO, NY 4182/1119, Bl. 55-57, und Heil, Verwaltungsgerichtsbarkeit, S. 69-71.

99 Siehe Protokoll der Beratung der Genossen Fechner/Ulbricht mit den Genossen Fischer, Warnke, Bechler, Siewert und mit dem Präsidenten der Deutschen Verwaltung für Inneres, 20.12. 1946, SAPMO, NY 4182/1192, Bl. 188-194, hier 193f. Teildruck des Protokolls, nicht aber des Abschnitts über die Verwaltungsgerichtsbarkeit, in Amos, Justizverwaltung, S. 224-226.

100 Siehe dazu Schäfermeyer in: Stenographische Niederschrift über dic Sitzung des Ausschusses für Rechtsfragen am 21./22. 6. 1947, SAPMO, DY 30 IV 2/1.01/51, Bl. 119. Ein Gesetzesentwurf aus der SED-Justizabteilung vom November 1946 in: SAPMO, NY 4182/1119, Bl. 62-69.

101 Vermerk Langes über eine Unterredung von Melsheimer und Meyer mit Karassjow am 29. 3. 1947, BAB, DP1 VA Nr. 11, Bl. 107. 
ner zu erhoffenden interalliierten Einigung über die Grundsätze für die Wiederherstellung der deutschen Wirtschaftseinheit' aufzuschieben, ,um die Zäsur zwischen dem Rechtszustand hier und dem übrigen Deutschland nicht noch zu vertiefen'“102. Die SMAD-Rechtsabteilung gestand schließlich Schiffer eine stark eingeschränkte Generalklausel zu ${ }^{103}$; dieser blieb jedoch bei seiner ablehnenden Haltung.

Auf der in dieser Situation anberaumten Beratung im SED-Ausschuß für Rechtsfragen am 21./22. Juni 1947 ging es erneut um die Alternative Generalklausel oder Enumerationsprinzip. Melsheimer sah die Diskussion angesichts der sowjetischen Entscheidung für die Generalklausel zwar als überflüssig an; Polak und Fechner erklärten jedoch selbstbewußt, daß die SED aus diesem Grund nicht auf einen eigenen Standpunkt verzichten müsse. Die Frage war auch unter den SED-Juristen noch durchaus strittig: Wieder trat Fritz Geyer aus Sachsen für das Enumerationsprinzip und Karl Schultes aus Thüringen für die Generalklausel ein. Zur Diskussion stand wieder die Frage, wie trotz der Errichtung von Verwaltungsgerichten deren Wirksamkeit am effektivsten beschnitten werden könne. War für Geyer die Einführung des Enumerationsprinzips conditio sine qua non, um zu verhindern, „daß die Verwaltungsgerichte an unserem Aufbau herumknabbern", so sah Schultes keine Gefahr in einer stark eingeschränkten Generalklausel: „Wenn man gesetzlich dafür sorgt, daß die Verwaltungsgerichtsbarkeit keine üblen Sprünge macht, ist es praktisch keine Generalklausel mehr." Fechner favorisierte demgegenüber das Enumerationsprinzip - aus Gründen der besseren Kosmetik: Es sei „undemokratisch“, bei einer Vielzahl von Legislativakten den Gang zum Verwaltungsgericht gesetzlich auszuschließen, da dadurch der Eindruck erweckt werde: „man will etwas verschweigen, die Rechte sollen wieder eingeengt werden usw." Gegen seinen Vorschlag, einen entsprechenden Zentralsekretariatsbeschluß herbeizuführen, erhob sich kein Widerspruch.

Die SMAD hatte jedoch, wie Melsheimer berichtete, Befehlsentwürfe vorbereitet, in denen die Generalklausel verankert war. Obwohl noch nicht endgültig klar war, daß die Verwaltungsgerichtsbarkeit auf dem Befehlsweg oder über Landesgesetze eingeführt werden sollte, wollte Fechner sich umgehend „der Sache annehmen" ${ }^{104}$. Bereits zwei Tage nach der Sitzung, am 24. Juni, beschloß das Zentralsekretariat einer von Fechner vorgelegten Stellungnahme zuzustimmen, „die Generalklausel abzulehnen und bei weiteren Verhandlungen die Enummeration [sic] zugrundezulegen"105. Daß Fechner nun in Verhandlungen mit Karlshorst eintrat und dort mit seinem Anliegen auf offene Ohren stieß, ist zwar nicht belegt, aber sehr wahrscheinlich. Denn nur so ist die Bestürzung in der SED-Justizabteilung auf die telefonische Mitteilung der SMAD-Rechtsabteilung vom 1. Juli erklärbar, „daß offenbar seitens der höchsten Leitung in Karlshorst entschieden worden sei, die Frage der Verwaltungsgerichtsbarkeit durch die Landtage und nicht durch

102 Heil, Verwaltungsgerichtsbarkeit, S. 171 f. Das Zitat S. 171.

103 Mitteilung Melsheimers auf der Sitzung des Ausschusses für Rechtsfragen, 21./22.6. 1947, SAPMO, DY 30 IV 2/1.01/51, Bl. 122.

104 Ebenda, Bl. 119-123. Zu dieser Diskussion vgl. auch Amos, Justizverwaltung, S. 191. Heil, Verwaltungsgerichtsbarkeit, S. 169, geht nur knapp auf die Diskussion auf der Grundlage eines Kurzprotokolls ein (siehe: BAB, DP1 VA Nr. 14).

105 Protokoll der Zentralsekretariatssitzung, 24. 6. 1947, SAPMO, DY 30 IV 2/2.1/104, TOP 6. 
Zonenbefehl ergehen zu lassen“. Polak und Schäfermeyer hielten dies „für außerordentlich ungünstig“, da sie mit erheblichem Widerstand von CDU und LDP gegen das Enumerationsprinzip rechneten ${ }^{106}$. Es war dann auch nur ein scheinbarer Widerspruch zu dieser Ankündigung, daß am 8. Juli die SMAD doch einen Befehl über die Verwaltungsgerichtsbarkeit erließ. Denn darin forderte sie die Ministerpräsidenten von Mecklenburg, Brandenburg, Sachsen und der Provinz Sachsen lediglich auf, den Entwurf eines Verwaltungsgerichtsgesetzes den Landtagen zur Beratung vorzulegen; ab dem 1 . Oktober sollten die Gerichte tätig werden ${ }^{107}$. Damit hatte sich Karlshorst letztlich einer verbindlichen Anweisung entzogen, ermöglichte aber der SED über die Koordinierung des Gesetzgebungsverfahrens maßgeblichen Einfluß auf die Gestaltung der Verwaltungsgerichtsbarkeit zu nehmen. $\mathrm{Da}$ die Militärverwaltungen in den einzelnen Ländern mit der Kontrolle über die Ausführung des Befehls betraut wurden, behielt die sowjetische Seite auch weiterhin die Möglichkeit eines korrigierenden Eingriffs, falls sich dabei das Blatt zugunsten der bürgerlichen Parteien wenden sollte.

In der SED-Zentrale nahm man diese Aufgabe umgehend in Angriff, indem Polak, Fechner und Anton Plenikowski je einen Vertreter der Landesinnenministerien und der SED-Fraktionen aus den Rechtsausschüssen der Landtage, einen Genossen vom Berliner SED-Landesvorstand und Melsheimer zu einer Unterredung am 26. Juli einluden. Bei dieser Gelegenheit wurde ein Entwurf ausgearbeitet, der von den SED-Fraktionen in den Länderparlamenten eingebracht werden sollte ${ }^{108}$. Nachdem die Innenministerkonferenz diesem Verfahren am 11./12. August zugestimmt hatte ${ }^{109}$, versandte das SED-Zentralsekretariat am 1. September den „Entwurf zur Ausführungsverordnung des Kontrollratsgesetzes Nr. 36“ zusammen mit einer ausführlichen Erläuterung an die SED-Landesvorstände. Darin wurde nochmals in deutlichen Worten der SED-Standpunkt dargelegt: $\mathrm{Da}$ an der Errichtung von Verwaltungsgerichten kein Weg vorbeiführe, müsse vermieden werden, daß diese rechtlich „zu einer Waffe der Reaktion gegen die demokratischen Verwaltungsorgane" würden. Dazu wurden in den Verordnungsentwurf die bekannten Sicherungen - insbesondere Wahl und Absetzbarkeit der Verwaltungsrichter, Enumerationsprinzip und Ausschluß der Verwaltungsgerichtsbarkeit bei sogenannten "Regierungsakten“ - eingebaut, um Regierung und Verwaltung möglichst wenig zu beeinträchtigen ${ }^{110}$. Von einem ernsthaften Rechtsschutz konnte jedenfalls keine Rede mehr sein.

Im Potsdamer Landtag wurde der SED-Entwurf am 9. Oktober aufgrund sowjetischen Drucks ohne nennenswerte Diskussion angenommen ${ }^{111}$. In Schwerin brachte die CDU, in Dresden die LDP einen eigenen Gesetzesentwurf ein. In bei-

106 Abtcilung Justiz an Ulbricht/Fechner, 1. 7. 1947, SAPMO NY 4182/1120, Bl. 56. Vgl. auch Amos, Justizverwaltung, S. $191 \mathrm{f}$.

107 Befehl Nr. 173 in: BAB, DX1, Sammlung SMAD-Befehle. Der Inhalt ist bereits korrekt wiedergegeben in: Meyer, Entwicklung, S. 562.

108 Abteilung Landespolitik an Ulbricht/Fechner, Betr.: Tagesordnung für das Treffen am 11. und 12. 8. 1947, o.D., SAPMO DY 30 IV 2/13/109, Bl. 49.

109 Protokoll der Innenminister-Konferenz am 11./12. 8. 1947, ebenda, Bl. 50-57, hier 55.

110 SED-Zentralsekretariat an alle SED-Landesvorstände, 1. 9. 1947, SAPMO, NY 4182/1090, Bl. 43;

Verordnungsentwurf Bl. 52-59; Erläuterung Bl. 60-65. Schreiben und Erläuterung auch in: Amos, Justizverwaltung, S. 276-281.

111 Vgl. Pohl, Justiz in Brandenburg (Manuskript), S. 54. 
den Fällen konnte die SED jedoch die bürgerlichen Blockparteien dazu bewegen, der SED-Vorlage zuzustimmen, so daß das mecklenburgische Gesetz am 18. September und das sächsische am 30 . Oktober 1947 verabschiedet wurden ${ }^{112}$. Da im Landtag in Halle „eine Einigung über Art und Umfang des Verwaltungsrechtsschutzes weder zwischen den Parteien noch in der SED-Fraktion erzielt werden konnte “ ${ }^{113}$, blieb Sachsen-Anhalt zunächst ohne ein Verwaltungsgerichtsgesetz. Verwaltungsgerichte wurden schließlich nur in Mecklenburg und, nach langem Hin und Her, in Brandenburg errichtet ${ }^{114}$. Der sächsische Landtag wählte lediglich Kurt Ebert im April 1948 zum Landesverwaltungsgerichtspräsidenten ${ }^{15}$. Im September 1949 und Mai 1950 - zwei Jahre nach Verabschiedung des Gesetzes erließ der Landtag zwar die für die Anwendbarkeit des Enumerationsprinzips notwendigen Ausführungsregelungen; ein Verwaltungsgericht wurde in Sachsen jedoch nicht tätig16. Der Landtag in Sachsen-Anhalt beschloß im Juni 1950 ein entsprechendes Gesetz; da jedoch die erforderliche Ausführungsverordnung ausblieb, kam es auch hier nicht zur Bildung eines Verwaltungsgerichts ${ }^{117}$. Thüringen war insofern ein Sonderfall, als es 1947 bereits über ein Verwaltungsgericht verfügte, so daß SMAD-Befehl Nr. 173 sich nicht an den Weimarer Ministerpräsidenten richtete. Dennoch wurde auf Betreiben der SED auch im thüringischen Landtag - freilich mit einjähriger Verzögerung - ein Verwaltungsgerichtsgesetz nach ihren Vorstellungen verabschiedet ${ }^{118}$.

Im Unterschied zu den Westzonen 119 wurde in der SBZ nur eine äußerst rudimentäre Verwaltungsgerichtsbarkeit wiederhergestellt. Deren Wirksamkeit war, sieht man einmal von der Tätigkeit des Thüringer Oberverwaltungsgerichts ${ }^{120} \mathrm{ab}$, äußerst begrenzt. Die 1947/48 ins Leben gerufenen Landesverwaltungsgerichte blieben zwar noch bis zur Auflösung der Länder im Jahre 1952 bestehen und konnten Bürger in Einzelfällen auch vor rechtswidrigen Verwaltungsakten schützen $^{121}$, stellten jedoch aufgrund ihrer stark eingeschränkten Zuständigkeit kein Hindernis beim „Aufbau des Sozialismus“ dar. Im Zuge ihrer Wiedereinführung war verhindert worden, daß mit den Verwaltungsgerichten auch der vornationalsozialistische Rechtsschutz im vollen Umfang wiederhergestellt wurde. Denn die SMAD-Rechtsabteilung verwarf im April 1947 die Vorstellungen der DJV, die die

112 Zur Diskussion in den Landtagen siehe Anders, Demokratisierung, S. 142-144. Die Gesetze in: Gesetz- und Verordnungsblatt der Landesregierung Brandenburg 1947, S. $27-30$ (12. 10. 1947); Regierungsblatt für Mecklenburg 1947, S. 250-252; Gesetze/Befehle, Verordnungen, Bekanntmachungen veröffentlicht durch die Landesregierung Sachsen, 1947, S. 509-511.

113 So Heil, Verwaltungsgerichtsbarkeit, S. 174.

${ }_{114} \mathrm{Zu}$ Brandenburg vgl. Pohl, Justiz in Brandenburg (Manuskript), S. $54 \mathrm{f}$.

115 Vgl. Zieger/Zieger, Verfassungsentwicklung, S. 104; Lebenslauf Eberts, 15. 11. 1951, BAB, DP1 SE Nr. 673. Im Mai 1950 trat Ebert von diesem Amt zurück.

116 Vgl. Brunner, Verwaltungsgerichtsbarkeit, S. 41-43.

117 Heil, Verwaltungsgerichtsbarkeit, S. 175.

$118 \mathrm{Zu}$ den Hintergründen für die verzögerte Übernahme in Thüringen ebenda, S. 175-177, 197-236; Weber, Justiz und Diktatur, S. 74-78; Loening, Kampf, passim; das Gesetz über die Verwaltungsgerichtsbarkeit, 7. 10. 1948, in: Regierungsblatt für das Land Thüringen 1948 I, S. 103-105.

119 Vgl. dazu Ule, Geschichtliche Entwicklung, S. 81-88.

120 Aber auch Thüringen war bis 1948, im Gegensatz zur Auffassung Heils, keine "Oase der Verwaltungsgerichtsbarkeit“, da alle Maßnahmen der SED zur Durchsetzung ihrer wirtschafts- und gesellschaftspolitischen Ziele der Verwaltungsrechtsprechung entzogen waren: vgl. Weber, Justiz und Diktatur, S. 59-70.

121 Vgl. Janke, Verwaltungsgerichtsbarkeit, S. $429 \mathrm{f}$. 
Zuständigkeit der Verwaltungsgerichte mit einer kaum beschränkten Generalklausel regeln wollte. Sie bevorzugte den ihr ebenfalls vorliegenden Gesetzesentwurf der SED-Justizabteilung, die sich in diesem Zusammenhang bereits sehr früh engagierte ${ }^{122}$, und schaltete damit die DJV aus der Diskussion aus. Die SED-Führung und der Parteiapparat erhielten dadurch die Möglichkeit, maßgebenden Einfluß auf die Formulierung der Verwaltungsgerichtsgesetze zu nehmen. Zunächst rechnete die SED-Justizabteilung offensichtlich damit, daß die SMAD per Befehl das Enumerationsprinzip verankern würde; als der Befehl aus Karlshorst die $\mathrm{Zu}$ ständigkeitsfrage offen ließ, nutzte die SED-Führung die Möglichkeit, über die Koordinierung des Gesetzgebungsverfahrens ihre Vorstellungen umzusetzen. Im Unterschied zur Einführung der Kassation war die SED bei der Beschneidung der Wirksamkeit der Verwaltungsgerichtsbarkeit somit nicht nur durchführendes Organ der SMAD, sondern auch mitgestaltende Kraft.

\section{Die SMAD-Rechtsabteilung und die Kontrolle der Rechtsprechung durch die DJV}

Neben Eingriffen in das Normensystem versuchte die SMAD-Rechtsabteilung über die DJV auch die Strafrechtsprechung zu kontrollieren und zu beeinflussen. Schiffer hielt es ebenfalls für eine Pflicht der DJV, die Gerichtstätigkeit sowohl durch Auswertung von statistischem Material als auch durch Revisionen vor Ort zu überwachen, und begründete dies vor allem mit dem Einsatz „neuer und unerfahrener Kräfte in der Rechtspflege“. Des weiteren müsse die DJV „einheitliche Richtlinien für die Praxis der Gerichte" geben; dies beinhaltete freilich keine Maßnahmen, die die Unabhängigkeit der Gerichte berührten, sondern erstreckte sich lediglich auf die Vorgabe bestimmter Fristen für den Rechtsgang, die Beachtung gleicher Grundsätze im Strafvollzug und ein einheitliches Gnadenverfahren ${ }^{123}$. Dementsprechend allgemein waren die Aufgabenbeschreibungen der Abteilung III, derzufolge die Überwachung der Gerichtstätigkeit „unbeschadet der Unabhängigkeit der Gerichte“ umfassend und „auf Sicherung und Verankerung des neuen demokratischen Staates" ausgerichtet sein müsse ${ }^{124}$. Dabei ging es Abteilungsleiter Corsing wohl vor allem darum, einen Rückfall in die Gerichtspraxis vor $1945 \mathrm{zu}$ vermeiden.

Daß die SMAD-Rechtsabteilung ebenfalls großen Wert auf diese Aufgabe legte, zeigt ein Schreiben Karassjows vom November 1945: „Größte Bedeutung hat im gegenwärtigen Moment die Frage der Überprüfung der Tätigkeit der Gerichte. Wie verwirklichen die reorganisierten Gerichte in der Praxis die demokratischen Prinzipien der Rechtspflege?"125 Diese Bemerkungen konnten von Schiffer, der sich dem Ziel eines demokratischen Rechtsstaats verpflichtet fühlte, als Bestäti-

122 Siehe dazu neben den Interventionen in den "Gesetzgebungsprozeß" auch die Artikel von zwei Mitgliedern der SED-Justizabteilung: Berger, Verwaltungsgerichtsbarkeit, sowie Schäfermeyer, Sinn.

${ }^{123}$ Chef der DJV an Chef der SMAD-Rechtsabteilung, 30. 10. 1945, BAB, DP1 VA Nr. 2, Bl. $94 \mathrm{f}$.

124 Aufgabenkreis des Direktors Dr. Corsing, o.D. [Oktober 1945], BAB, DP1 VA Nr. 1043, Bl. 10.

125 Chef der SMAD-Rechtsabteilung an Chef der DJV, 14. 11. 1945, BAB, DP1 VA Nr. 2, Bl. 103. 
gung seines Kurses aufgefaßt werden. Die SMAD-Rechtsabteilung setzte bei ihren Mahnungen zur Intensivierung der Gerichtskontrolle ${ }^{126}$ jedoch einen etwas anderen Akzent als die Justizverwaltung. Denn als Karassjow am 8. Juni 1946 forderte, „daß die Zentrale Justizverwaltung sich noch stärker als bisher der Führung der Rechtsprechung der neuen Gerichte annehmen müsse“, gab er auch die Richtung vor, in die die DJV die Rechtsprechung lenken sollte: Die Richter müßten „sich dessen bewußt sein, daß sie nicht nur eine isolierte Funktion auszuüben hätten, [...] sondern daß auch sie nur einen Teil des großen Leitungs- und Führungsapparates der Gesellschaft darstellten [... und] mit ihrer Urteilstätigkeit an der Förderung der allgemeinen Gesellschaftszwecke mitzuwirken hätten“. Die Justiz hatte folglich für die sowjetische Militärverwaltung vor allem einen instrumentellen Charakter. Daher ermahnte Karassjow die DJV, „sich insbesondere ein Bild davon zu machen, welche Richter und Gerichte in diesem Sinne gute Urteile sprechen, und welche Richter und Gerichte versagten"127. Mit anderen Worten: Die DJV sollte die Richter mittels positiver und negativer Beispiele zu einer ,fortschrittlichen Rechtsprechung' erziehen. Über eine derartige, die richterliche Unabhängigkeit beeinträchtigende Anordnung der DJV ist freilich nichts bekannt.

Ihre Unzufriedenheit mit der Strafrechtspflege in der SBZ und deren mangelhafter Kontrolle durch die DJV bekundete die SMAD-Rechtsabteilung im Jahre 1946 mehrfach. Richtete sich die sowjetische Kritik im Mai gegen die Gestaltung der Quartalsberichte über die Arbeit der Gerichte und Staatsanwaltschaften - die Rechtsabteilung vermißte darin eine „Analyse der grundlegenden Fragen der richterlich-staatsanwaltschaftlichen Tätigkeit und konkrete Maßnahmen für ihre Verbesserung " 128 - war es am 31. Juli eine große Anzahl von - nicht näher bezeichneten - Mißständen und falschen Urteilen in Brandenburg, die den Unwillen Karassjows hervorrief. Besonders verärgert war er darüber, daß einige beanstandete Gerichte von der DJV überprüft, aber nicht zur Abstellung der Mängel angewiesen worden seien. Er ermahnte Schiffer daher, die Revisionstätigkeit zu verstärken und „zur Beseitigung der Mißstände [...] die energischsten Maßnahmen“ zu treffen ${ }^{129}$. Anscheinend trat daraufhin - zumindest nach Auffassung der SMAD keine entscheidende Besserung ein, so daß anläßlich eines Vortrags der Leiter der DJV-Abteilungen III und IV in Karlshorst am 11. September 1946 die SMADRechtsabteilung erneut die Kontrolltätigkeit der DJV scharf kritisierte ${ }^{130}$. Jetzt erst reagierte die DJV, indem sie am 19. September die Kritik an die Landesjustizabteilungen per Rundschreiben weitergab: Die Strafrechtsprechung der vergangenen zwölf Monate hätten „noch nicht den an sie zu stellenden Anforderungen genügt“", und die Gerichte hätten in einigen Fällen - bei schweren Beleidigungen der

126 Vgl. auch Aktenvermerk über Unterredung Schiffers mit Nikolajew am 8. 1. 1946, BAB, DP1 VA Nr. 11, Bl. 5.

${ }^{127}$ Kleikamp an Schiffer, 19.6. 1946, betr.: Rücksprache in Karlshorst zwischen Kanger, Kleikamp, Karassjow und Lyssjak am 8. 6. 1946, ebenda, Bl. 53.

128 Aktenvermerk über die Rücksprache Schiffers mit Karassjow am 17.5. 1946, ebenda, Bl. 41; SMAD-Rechtsabteilung an Chef der DJV, 18. 5. 1946, BAB, DP1 VA Nr. 2, Bl. 227-230.

${ }_{129}$ Aktenvermerk über den Besuch Schiffers in Karlshorst am 31.7. 1946, BAB, DP1 VA Nr. 11, Bl. 62.

130 Siehe dazu den zusammenfassenden Bericht über die Revision der Gerichtsbehörden, insbesondere der Strafrechtspflege, 31. 12. 1946, BAB, DP1 VA Nr. 12, und Chef der DJV an Chef der SMAD-Rechtsabteilung, 28. 9. 1946, BAB, DP1 VA Nr. 2, Bl. 320. 
Besatzungsmacht und Waffenbesitz - so milde Urteile gefällt, daß der Abschrekkungszweck der Strafe nicht erreicht worden sei. Zur besseren Kontrolle der Strafrechtspflege ordnete Schiffer daher eine Ausweitung der Berichtspflichten der Justizabteilungen an. Während diese bisher nur über Sabotagefälle, Preisstrafverfahren und Strafsachen, in denen auf Todesstrafe erkannt wurde, berichten mußten, kamen nun Verfahren mit politischem Einschlag, Verfahren wegen Verbrechen gegen die Menschlichkeit (gemäß Kontrollratsgesetz Nr. 10131) und die Verfahren hinzu, die in der Öffentlichkeit Aufsehen erregt hatten oder in der Presse kritisiert worden waren. Des weiteren kündigte er „eine eingehendere und häufigere Revision der Gerichte, insbesondere der Strafgerichte, und Staatsanwaltschaften" durch die DJV an und wies auch die Justizabteilungen an, Inspektionen durchzuführen, entdeckte Mängel durch Belehrungen abzustellen und "erforderlichenfalls" sogar ungeeignete Richter und Staatsanwälte zu entlassen ${ }^{132}$.

Bei den geplanten Überprüfungen, so schrieb er am 28. September an Karassjow, werde es primär um die Prüfung der Frage gehen, ,in welchem Geist die Aufgaben der Justiz gelöst“" würden. „Dieser Geist“, so Schiffer weiter, „muß frei sein von allen faschistischen und militaristischen Vorstellungen. Dieser Geist muß mit allen überkommenen Gedankengängen reaktionärer, formalistischer und bürokratischer Art brechen." Die Revisionen würden unter anderem eine Überprüfung sämtlicher Strafurteile eines bestimmten Zeitraums nach Straftat, Motiv, angewandtem Strafgesetz, Strafantrag, Strafmaß und Gründen für die Strafzumessung, die Feststellung der Verfahrensdauer und die Teilnahme an den Sitzungen umfassen. In einer ausführlichen Abschlußbesprechung werde nicht nur auf Fehler und Mängel, sondern auch „auf die nachdrückliche Bekämpfung besonders wichtiger Straftaten (Sabotage, Bandendiebstähle, Wirtschaftsvergehen, Jugendstrafsachen)“, auf eine sorgfältige Statistik, auf die Notwendigkeit einer engen Zusammenarbeit mit Polizei, Wirtschaftsbehörden und Jugendämtern und darauf hingewiesen, daß von der richterlichen Tätigkeit „die Entwicklung des demokratischen Gedankens in hohem Maße abhängt". Wenngleich sich damit Möglichkeiten der Einwirkung auf die richterliche Tätigkeit ergaben, war Schiffer von dem instrumentellen Rechtsverständnis der SMAD-Rechtsabteilung weit entfernt. Vielmehr wollte er auch durch Revisionen die Justizjuristen auf ihre „Verpflichtung zur Herstellung einer wahrhaft volkstümlichen Rechtspflege" hinweisen, um seinem Reformziel, der Überwindung der Kluft zwischen Volk und Recht, näherzukommen ${ }^{133}$.

Einer verstärkten Überwachung der Gerichte - sei es unmittelbar durch die DJV, sei es mittelbar durch die Justizabteilungen - stand 1946/47 jedoch eine Reihe von Hindernissen entgegen. Aus Sicht der Zentralverwaltung hatten in diesem Zusammenhang die Landesjustizverwaltungen versagt ${ }^{134}$. Rosenthal-Pelldram stellte am 19. November 1946 fest, daß vor allem die Justizabteilungen in

131 Kontrollratsgesetz Nr. 10, 20. 12. 1945, in: Amtsblatt des Kontrollrats, Nr. 3, 31. 1. 1946, S. 50-55.

Dies war die einzige Rechtsgrundlage, auf der deutsche Gerichte damals NS-Verbrechen bestrafen konnten: siehe dazu Kap. B.VII.1.

132 DJV an Landes- und Provinzialregierungen/Abteilung Justiz, 19. 9. 1946, BAB, DP1 VA Nr. 2, Bl. $318 \mathrm{f}$.

133 Chef der DJV an Chef der SMAD-Rechtsabteilung, 28. 9. 1946, ebenda, Bl. 320-323.

134 So Rosenthal-Pelldram in der Dienstbesprechung vom 16. 10. 1946, BAB, DP1 VA Nr. 7354. 
Weimar, Halle und Potsdam sich zu wenig um die Gerichte und Staatsanwaltschaften kümmerten, "und nicht umsonst würden gerade in diesen Teilen der sowjetischen Besatzungszone die Revisionen der Deutschen Justizverwaltung begrüßt“. Auch Schiffer bezeichnete die Zusammenarbeit mit den Ländern und Provinzen, die nicht nur aus Personalmangel, sondern auch aus „einem Mangel an Verständnis" unzureichendes und lückenhaftes Material zur Verfügung stellten, als „nicht befriedigend“"135. Im März 1947 bezeichnete der neue Leiter der Abteilung III, Löwenthal, die Mitarbeit der Landesjustizverwaltungen zwar als „nicht schlecht", aber keineswegs als optimal und kritisierte, daß die DJV nicht direkt mit den Staatsanwaltschaften und Gerichten verkehren könne ${ }^{136}$. Des weiteren reichte das Personal der DJV für eine Intensivierung der Revisionstätigkeit bei weitem nicht aus. Im Oktober 1946 war der Leiter der zuständigen Abteilung, Corsing, aus der DJV ausgeschieden und der einzige Referent der Abteilung, Hartwig, vorläufig mit deren Leitung betraut worden ${ }^{137}$. Da ihn lediglich ein Sachbearbeiter und ein Registrator unterstützten, wurden auch andere Abteilungs- und Referatsleiter der DJV zu den Revisionen herangezogen ${ }^{138}$. Anfang 1947 verbesserte sich die Personalsituation in der Abteilung zwar durch die Zuteilung von zwei Referenten ${ }^{139}$ und durch die Ernennung von Löwenthal zum Abteilungsleiter ${ }^{140}$, dennoch beklagte sich dieser im Mai über die zu schwache Besetzung, die zwar für die Kontrolle der Justizbehörden, nicht aber auch noch für die Auswertung der Ergebnisse ausreiche ${ }^{141}$. Schließlich dürfen die technischen Schwierigkeiten nicht außer Acht gelassen werden: Die für Mitte Dezember 1946 vorgesehene Revision der Gerichtsbehörden in Zwickau etwa konnte aufgrund eines Achsenbruchs des Dienstwagens nicht durchgeführt werden. Zum schlechten Zustand der Kraftwagen kamen Benzinmangel, die winterliche Witterung und die Einschränkung des Eisenbahnverkehrs hinzu, so daß im Dezember 1946 und im Januar 1947 keine Inspektionsfahrten unternommen werden konnten ${ }^{142}$.

Trotz der ungünstigen Umstände führte die DJV von Ende September bis Ende November 1946 Revisionen der Justizbehörden in insgesamt 14 Orten durch. Die Bilanz dieser Überprüfungen, die Hartwig in einem für die SMAD-Rechtsabteilung gedachten Bericht am 31. Dezember 1946 zog, zeigt, daß es damals weder um eine im sowjetischen Sinne „fortschrittliche oder „rückschrittliche“ Rechtsprechung, noch um eine Justizreform im Sinne Schiffers gehen konnte, sondern schlichtweg darum, die Gerichtsbarkeit in ihren Grundfunktionen zu sichern. Die Masse der Beanstandungen war auf die ungünstigen Rahmenbedingungen zu-

135 Vermerk über die Besprechung vom 19.11. 1946, ebenda.

136 Vermerk über die Dienstbesprechung vom 13. 3. 1947, ebenda.

137 Siehe Kap. A.I.4.

${ }^{138}$ Laut Bericht vom 31.12. 1946 waren die Revisionen neben Hartwig von Rosenthal-Pelldram, Hirschfeld, Benjamin, Weiß, Lentz, Winkelmann, und Meyer durchgeführt worden, BAB, DP1 VA Nr. 12.

${ }^{139}$ Hermann Hirschfeld und Bernhard Schreiber: siehe Verfügung Schiffers, 13. 1. 1947, BAB DP1 VA Nr. 5777.

140 Siehe Kap. A.I.4.

${ }_{141}$ Vermerk über die Dienstbesprechung vom 16. 5. 1947, BAB, DP1 VA Nr. 7354.

142 Siehe den zusammenfassenden Bericht über die Revision der Gerichtsbehörden, insbesondere der Strafrechtspflege, 31.12. 1946, BAB, DP1 VA Nr. 12, und Vermerk über die Besprechung vom 22. 1. 1947, BAB, DP1 VA Nr. 7354. 
rückzuführen, unter denen die Justiz in der SBZ existierte. Erstens arbeite das Personal zwar "mit Eifer und Hingebung", reiche aber weder quantitativ noch qualitativ aus. Dabei gab es Hartwig zufolge auch unter den Volljuristen unzureichende Kräfte, die teilweise dem Richterberuf durch lange Untätigkeit entfremdet waren, teilweise aus anderen Rechtsgebieten wie dem Sudetenland kamen oder als Anwälte "nicht mehr die richtige Einstellung zum Richterberuf“ fanden. Unter den - damals freilich kaum tätigen - Absolventen der Richterschulen seien demgegenüber einige, die „ihren Platz zur vollen Zufriedenheit“ ausfüllten. Zweitens fielen "nicht unerhebliche Abweichungen hinsichtlich der Rechtsanwendung" zwischen den einzelnen Regionen ins Gewicht, die nicht nur auf einer unterschiedlichen Rechtsauslegung, sondern auch „auf der selbständigen Rechtsschöpfung der Länder und Provinzen“ beruhten. Drittens waren zahlreiche Defizite in der Strafrechtspflege auf Probleme zurückzuführen, für die die Justiz letztlich nicht verantwortlich war: Hartwig nannte unter anderem den großen Mangel an Gerichtsgefängnissen, die häufige Beteiligung von Ausländern an Straftaten sowie die unzulängliche Ausbildung der Kriminalpolizei. An den Verfahren kritisierte er die zu lange Dauer der Untersuchungshaft und die zu häufige Anwendung des Schnellverfahrens. Die Durchsicht der Strafakten schließlich ergab neben der Feststellung technischer Fehler „vielfach eine ganz unzureichende Begründung des Strafmaßes: Es war zwar auf die zu Gunsten des Angeklagten sprechenden Milderungsgründe, wie wirtschaftliche Notlage, hingewiesen worden; man vermißte aber ein Eingehen auf die Volksschädlichkeit der begangenen Straftat und die Betonung der Notwendigkeit einer strengen Bestrafung im Interesse der Abschreckung" ${ }^{143}$. Mit dieser Feststellung stand Hartwig nicht allein; auch Rosenthal-Pelldram konstatierte bei anderer Gelegenheit die „Tendenz zur Milde bei den Gerichten zur Zeit der Wiedereinrichtung der Gerichtsbarkeit" ${ }^{* 144}$.

Vertreter der SMAD-Rechtsabteilung bemängelten ebenfalls die generell $\mathrm{zu}$ milden Urteile der Strafrechtsprechung. Nikolajew etwa folgerte aus der geringen Beschäftigung der Oberlandesgerichte auf dem Gebiet des Strafrechts, „daß die Mehrzahl der Strafen zu milde sei; anderenfalls würden die Angeklagten in weit höherem Maße von der Möglichkeit eines Rechtsmittels Gebrauch machen"145. Auf der Länderkonferenz am 11. April 1947 rügte Jakupow, „daß die Straftätigkeit der Gerichte bei den am meisten verbreiteten Verbrechen sich durch eine übergroße Milde gegenüber den Verbrechern" ausweise und daß in der zweiten Instanz diese „liberalen Urteile“ häufig nochmals gemildert würden. „Diese Gerichte unter der Maske der Unabhängigkeit des Gerichts [sic]“, so führte er weiter aus, „bestärken bloß die Verbrecher durch ihre liberalen Urteile, aber helfen nicht der allgemeinen Sache des Kampfes gegen die Kriminalität." ${ }^{146}$

${ }^{143}$ Bericht über die Revision der Gerichtsbehörden, insbesondere der Strafrechtspflege, 31. 12. 1946, BAB, DP1 VA Nr. 12, und Vermerk über die Besprechung vom 19.11. 1946, BAB, DP1 VA Nr. 7354.

144 Protokoll der Konferenz der Generalstaatsanwälte am 25./26. 4. 1947, BAB, DP1 VA Nr. 14, Bl. 60.

145 Aktenvermerk über die Rücksprache Nikolajews mit Melsheimer am 15. 3. 1947, BAB, DP1 VA Nr. 11, Bl. 105.

146 Stenographisches Protokoll der Länderkonferenz vom 11.4. 1947, BAB, DP1 VA Nr. 6958, Bl. 33-35. 
Wenngleich das Eintreten für harte Urteile nicht unbedingt als typisches Charakteristikum für die sowjetische Besatzungsherrschaft angesehen werden kann, ist doch auffällig, wie häufig und auf welch harsche Weise die SMAD-Rechtsabteilung die Rechtsprechung der deutschen Gerichte kritisierte und eine Abstellung der von ihr diagnostizierten Fehler verlangte. Trotz aller Bekundungen, die Unabhängigkeit der Justiz zu wahren, sah sie offensichtlich keine Probleme darin, die Zentralverwaltung zu einer eingehenderen Kontrolle der Gerichte und zu Anweisungen anzuhalten, die diese Unabhängigkeit beeinträchtigen mußten. Dahinter verbarg sich ein instrumentelles Rechtsverständnis, das von der DJV nicht geteilt wurde. Diese kam den Forderungen der SMAD-Rechtsabteilung entgegen, hoffte aber, die verstärkte Revisionstätigkeit im Sinne ihrer Reformvorstellungen nutzen zu können. Weder durch die intensivierten Kontrollen noch durch die Ausweitung der Berichtspflichten konnte freilich in der unmittelbaren Nachkriegszeit eine effektive, zentralisierte Justizsteuerung über die DJV in die Praxis umgesetzt werden. Die von der SMAD-Rechtsabteilung vorgegebene Richtung bot jedoch genügend Ansätze, an die man in einer Zeit, in der die Anfangsschwierigkeiten beim Wiederaufbau des Justizwesens überwunden und die Weichen deutlicher auf Zentralisierung gestellt wurden, anknüpfen konnte. 